\title{
The evolution of pore shape and orientation in plastically deforming metals: implications for macroscopic response and shear localization
}

\author{
Dawei Song, M. Agoras, P. Ponte Castañeda \\ Department of Mechanical Engineering and Applied Mechanics, \\ University of Pennsylvania, Philadelphia, PA 19104-6315, U.S.A.
}

\begin{abstract}
A constitutive model is proposed for the macroscopic response of porous plastic metals at finite strains. Besides taking into account the porosity evolution, which leads to pressure sensitivity and dilatant response, the model can also account for changes in the average shape and orientation of the pores by means of suitable microstructural variables which play the role of internal variables and serve to characterize the evolving anisotropy of the material. In particular, the model is used to determine the evolution of the average shape and orientation of the voids under simple shear loading, as well as to explore the concomitant implications for the macroscopic response and shear localization. The intrinsic effect of the void rotations is deduced from comparisons with corresponding results for pure shear loading (where the voids change shape, but undergo no rotation on average), and found to be significant. In addition, more general loading conditions, involving combined tension and shear, are considered, and the effect of the stress triaxiality is investigated. For such more general plane strain conditions, it is found that there is an abrupt transition in the localization strain at a certain value of the triaxiality of about 0.3 to 0.4 , with the localization strain dropping sharply both as the triaxiality increases, or decreases from this value. Furthermore, the results suggest that void rotations can dramatically enhance the susceptibility of the material to shear localization for a certain range of triaxiality values (between, approximately, 0.3 and 0.8$)$.
\end{abstract}

Keywords: Constitutive models, anisotropy, porous plasticity, ductile failure, shear bands 


\section{Introduction}

Ductile metals are known to contain random distributions of micro-voids, which are produced either as a consequence of the forming process itself (e.g., powder metallurgy, HIPPing), or which nucleate in the material from second-phase particles and eventually grow and coalesce leading to material failure (Tvergaard, 1990; Benzerga and Leblond, 2010). When such porous materials are subjected to finite strains, the size, shape and orientation of the voids, as well as the positions of the voids relative to each other, evolve with the deformation. This paper is concerned with the use of certain recently developed homogenization approaches (Agoras and Ponte Castañeda, 2013; Agoras and Ponte Castañeda, 2014) to describe the instantaneous macroscopic response of porous rigid plastic materials given the current state of the microstructure, as well as the evolution of the microstructure with the deformation, and its implications for failure through shear localization. In the present work, however, we focus on loading conditions involving macroscopic loads leading to significant void rotations (as well as changes in porosity and void shape).

It should be emphasized that over the years there have been several other approaches that have been proposed to model the behavior of porous ductile materials. Gurson (1977) made use of limit analysis for a spherical shell to propose an isotropic, pressure-sensitive plasticity model accounting for dilatant behavior. This model has been shown to be accurate for nearly hydrostatic loading conditions (i.e., for high stress triaxialities), but less so for deviatoric loadings and, in particular, cannot account for void shape and orientation changes leading to anisotropy development for such loadings. Yamamoto (1978) considered the effects of compressibility and porosity evolution on theoretical predictions for shear localization in porous media, and found that localization is facilitated by increasing triaxiality. In particular, these Gurson model predictions indicate that the material should become more resistant to failure by shear localization at low to intermediate stress triaxialities. However, this is in contradiction with some experimental observations (Bao and Wierzbicki, 2004; Barsoum and Faleskog, 2007), which suggest that lower triaxialities tend to facilitate failure by shear localization at least for a certain range of low to intermediate triaxialities (but see also Haltom et al., 2013). Motivated by these experimental results, Nahshon and Hutchinson (2008) have recently proposed a phenomenological modification of the constitutive model of 
Gurson (1977), consisting in a reinterpretation of the porosity evolution law as an isotropic damage evolution law to empirically account for the effects of the third invariant of the loading (Lode angle) on the material response at low triaxialities. By introducing suitable parameters and fitting them to appropriate experimental data, this approach has been successful in modeling certain features of material failure at low triaxialities (Xue et al., 2013). On the other hand, the homogenization models of interest in this work aim to be entirely predictive, by introducing suitable microstructural variables directly accounting for the changes in shape and orientation of the voids, and the associated changes in the overall anisotropy and instantaneous hardening of the material. While capturing this level of detail is certainly more challenging, the potential gains in terms of predictive capabilities could justify the added computational cost, which would still be much smaller than that required for full-field numerical simulations.

Additional approaches include micro-mechanical approaches attempting to generalize the work of Gurson (1977) by considering more general spheroidal and ellipsoidal void shapes (Gologanu et al., 1993; Madou and Leblond, 2012a). The advantage of these approaches is that they give accurate predictions for high-triaxiality loading conditions, but they are less general than the homogenization approaches, in particular, because it has not been possible to derive corresponding evolution equations for the void rotation by means of these analyses. In this sense, the homogenization approaches to be developed in this work are more general since they provide consistent estimates for the average strain rate and vorticity in the voids, which can in turn be used to generate self-consistent evolution equations for the average void shape and rotation (Ponte Castañeda and Zaidman, 1994; Kailasam and Ponte Castañeda, 1998). Recently, Madou and Leblond (2013a) and Madou et al. (2013b) have proposed a combined approach making use of the Gurson limit analysis approach to obtain accurate estimates for the yield surface at high triaxialities, and of the homogenization approach of Ponte Castañeda and Zaidman (1994) and Kailasam and Ponte Castañeda (1998), improved through numerical fitting to finite-element simulations of confocal shells, to model the evolution of the void shape and rotation in the porous materials at low triaxialities.

A third approach is to make use of full-field numerical simulations, such as the ones recently carried out by Srivastava and Needleman (2013) and Tvergaard (2012, 2014), build- 
ing on earlier work (e.g., Needleman, 1972; Tvergaard, 1981). These simulations typically assume periodicity of the microstructure so that the numerical calculation can be restricted to a unit cell of the microstructure. While this approach is expected to be more accurate than the approximate homogenization models of interest in this work, the assumption of periodicity of the microstructure is a limiting factor, the microstructures of actual porous metals normally being random. Furthermore, the numerical results for periodic distributions of voids show great sensitivity to the microstructural parameters, suggesting that accounting for the randomness of the porosity distribution may be crucial. In this sense, the homogenization estimates to be discussed here offer the capability of accounting for the random distribution of the voids by means of the two-point correlation functions for their centers, as well as changes in the average shape and orientation of the voids.

The first homogenization estimates accounting for the overall compressibility in the instantaneous response of porous viscoplastic solids were given by Ponte Castañeda and Willis (1988), making use of the nonlinear Hashin-Shtrikman-type variational approach of Talbot and Willis (1985). Improved estimates were obtained by Ponte Castañeda (1991) making use of a new variational approach for a linear comparison composite (LCC) (see also Willis, 1991, and Michel and Suquet, 1992 for derivations of these estimates by other methods). Ponte Castañeda and Zaidman (1994) made use of the LCC variational homogenization method of Ponte Castañeda (1991) to advance constitutive models for porous viscoplastic solids accounting for the evolution of the microstructure (i.e., porosity and average void shape) under general triaxial loading conditions. In that work, the changes in pore shape were found to have a significant effect on the macroscopic response of the material at low stress triaxialities. In particular, it was shown that shear localization could take place at low triaxialities by a void collapse mechanism - something that could not be accounted for by the Gurson-type models. By making use of the linear estimates of Ponte Castañeda and Willis (1995), this model was generalized to account for void-distribution effects (Kailasam et al., 1997), void rotations under general non-vanishing spin loadings (Kailasam and Ponte Castañeda, 1997), as well as strain-hardening and elasticity for the matrix phase (Kailasam et al., 2000; Aravas and Ponte Castañeda, 2004). The numerical implementation of these models in general purpose finite-element codes (e.g., ABAQUS) was considered by Aravas 
and Ponte Castañeda (2004).

While the predictions generated by these variational models have been found to be quite good for deviatoric loadings, where void shape changes are dominant, they become progressively less accurate with increasing stress triaxiality, especially for low porosities and high material nonlinearities (see Ponte Castañeda and Suquet, 1998 for more details). For this reason, several attempts have been made to obtain improved homogenization estimates for porous viscoplastic materials. In particular, building on the earlier work by Danas et al. (2008), Danas and Ponte Castañeda (2009a, 2009b) proposed an improved constitutive model for porous materials with evolving microstructures. The model was derived by making use of the more sophisticated "second-order" LCC procedure of Ponte Castañeda (2002), together with an ad hoc interpolation/extrapolation scheme, enforcing the exact agreement of the second-order model with the Gurson model for the special case of spherical/cylindrical voids subjected to purely hydrostatic loadings. The second-order model was found to deliver fairly accurate results for the macroscopic response in several comparisons with FEM and other exact results. The model was also found to predict the development of shear localization instabilities due to void collapse at small stress triaxialities (Danas and Ponte Castañeda, 2012), although some of the predictions may need to be corrected to account for the possibility of contact of the void faces (Hutchinson and Tvergaard, 2012). In addition, it should be mentioned that Idiart (2008) has developed estimates based on sequentially layered microstructures, which have the distinguishing feature of reproducing exactly the hydrostatic point for spherical shells.

In this work, we will pursue an alternative approach that is based entirely on the variational linear comparison homogenization procedure - albeit used in a novel incremental fashion (Ponte Castañeda, 2012). This approach can account for the expected non-uniformity of the properties of the matrix phase in the porous LCC - and leads to results that are also in complete agreement with the predictions of the Gurson approach for high triaxialities. Thus, Agoras and Ponte Castañeda (2013) have developed an incremental procedure for generating constitutive models for porous viscoplastic materials consisting of random "ellipsoidal" distributions of ellipsoidal voids, where the shape and orientation of the voids may be different from that of their distribution. The method makes use of the work of Agoras and Ponte 
Castañeda (2011) to provide estimates for a finite number $N$ of iterations of the macroscopic viscoplastic stress potential for porous materials with given, fixed microstructure. When the number of iterations is $N=1$, the method recovers exactly the predictions of the earlier variational linear comparison method (Ponte Castañeda, 1991), and leads to progressively more accurate estimates as the number of iterations $N$ is increased, especially for high triaxialities. In the limit as $N \rightarrow \infty$ the iterated estimates of Agoras and Ponte Castañeda (2013) recover identically those of Ponte Castañeda (2012), when the shape of the pores and the distribution are identical, and therefore they also recover the well-known exact result for purely hydrostatic loadings of the composite-sphere assemblage (Leblond et al., 1994) and the infinite-rank sequentially laminated microstructures (Idiart, 2008). In practice, however, it can be shown that a small number of iterations $(N \simeq 5-10)$ is sufficient to generate accurate results, which implies that the new iterated estimates are also relatively easy to compute. In recent work, Agoras and Ponte Castañeda (2014) have made use of the iterated variational procedure of Agoras and Ponte Castañeda (2013) to obtain consistent evolution equations for the average void shape under general triaxial, finite-strain loading conditions. In the present work, we propose to consider more general loading conditions incorporating the effects of void rotations due to shear-type loadings. This will be accomplished following similar developments in the context of the earlier variational procedure by Kailasam and Ponte Castañeda (1998) (see also Aravas and Ponte Castañeda, 2004), which make use of consistent homogenization estimates for the average strain-rate and vorticity in the porous phase to generate evolution equations for the void orientation, as well as the porosity and average shape of the pores. To bring out the significant effect of the pore shape and orientation, some results will be shown for the effective yield surfaces of porous plastic materials with various values of the pore aspect ratios and orientation angles. In addition, we will consider simple shear loading and investigate the effect of void rotations by comparisons with pure shear loadings, which result in void shape changes, but not in void rotations. Then, the model will be used to investigate the effect of the stress triaxiality on the macroscopic response and possible development of shear band instabilities in rigid-plastic porous materials with power-law strain hardening that are subjected to combined shear and tension under plane strain conditions. Our focus will be on the effect of the evolution of the pore 


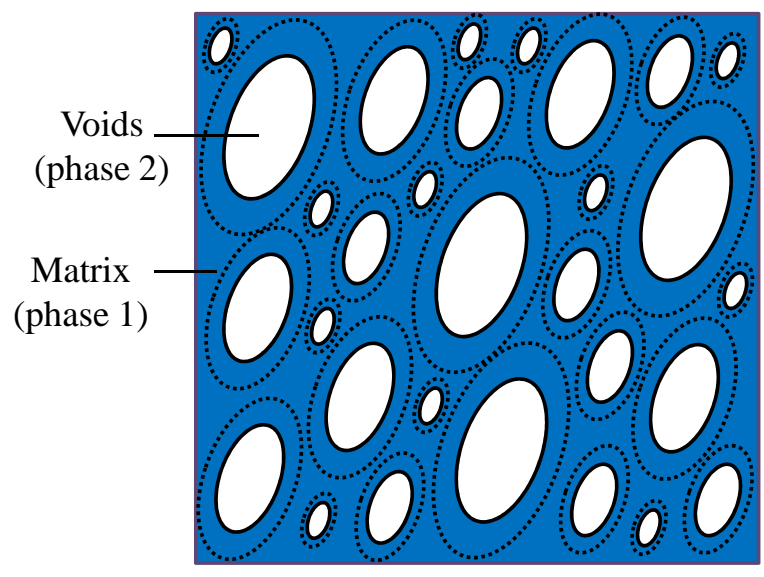

(a)

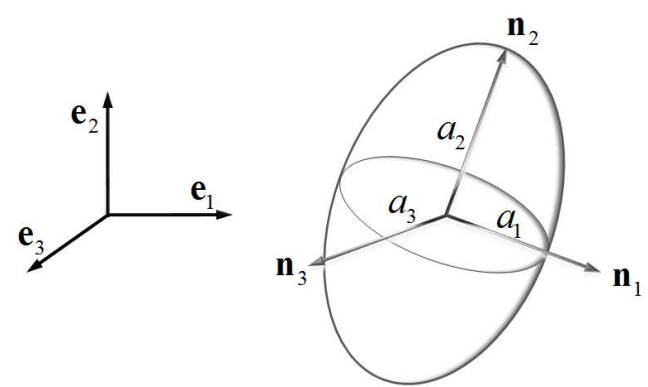

Geometrical Features of an Ellipsoid

(b)

Figure 1: Schematic representation of a porous metal consisting of aligned, ellipsoidal voids (solid lines) that are distributed with the same ellipsoidal symmetry (dotted lines) in a metal matrix.

orientation (and shape) on the anisotropic response and failure of the porous materials.

\section{The iterated variational linear comparison homogenization model}

In this section, we present the basic variables and equations of the Iterated Variational linear comparison Homogenization (IVH) model developed by Agoras and Ponte Castañeda (2013, 2014), suitably generalized to account for void rotations. We first introduce the internal variables of the IVH model, which characterize the hardening of the matrix (metal), porosity, shape and orientation of the voids. Next, we provide the macroscopic constitutive relation of the rigid-plastic porous metals and evolution laws for the above-mentioned internal variables. At last, expressions for the overall hardening rate are derived. For simplicity, elastic strains will be neglected in this work. However, it is straightforward to include such strains approximately, as was done, for example, by Aravas and Ponte Castañeda (2004).

\subsection{Internal variables}

The porous material is made up of two phases. The matrix material (phase 1) is isotropic, incompressible and rigid-plastic, and obeys the von Mises yield criterion:

$$
\Phi(\boldsymbol{\sigma})=\sigma_{e}^{2}-\sigma_{y}^{2}=0
$$


where $\sigma_{e}=\sqrt{\frac{3}{2} \boldsymbol{\sigma}_{d} \cdot \boldsymbol{\sigma}_{d}}$ is the equivalent stress $\left(\boldsymbol{\sigma}_{d}\right.$ is the stress deviator tensor) and $\sigma_{y}$ is the yield stress under uniaxial tension. In addition, isotropic hardening of the matrix is assumed so that $\sigma_{y}$ is a function of the accumulated plastic strain $\varepsilon_{M}^{p}$, such that

$$
\sigma_{y}\left(\varepsilon_{M}^{p}\right)=\sigma_{0}\left(1+\frac{\varepsilon_{M}^{p}}{\varepsilon_{0}}\right)^{M},
$$

where $\sigma_{0}$ is the initial tensile yield stress, $\varepsilon_{0}$ is the reference yield strain, and $M$ is the strain hardening exponent of the matrix.

As shown in Fig. 1, the voids (phase 2) are assumed to be of ellipsoidal shape (on average), and to be aligned, but distributed randomly in the matrix with a two-point correlation function for their centers characterized by "ellipsoidal" symmetry (Willis, 1977; Ponte Castañeda and Willis, 1995). In this work, for simplicity (Agoras and Ponte Castañeda, 2014), it is further assumed that the ellipsoidal shape and orientation of the distribution (i.e., the dashed ellipsoids in Fig.1) is identical to the ellipsoidal shape and orientation of the voids (i.e., the solid ellipsoids in Fig.1). However, it should be emphasized that, in general, the ellipsoid characterizing the distribution can be different from the ellipsoid characterizing the voids (Ponte Castañeda and Willis, 1995; Agoras and Ponte Castañeda, 2013).

According to the above hypothesis, the porous metal can be completely described by the set of internal variables defined by

$$
\mathbf{s} \equiv\left\{\varepsilon_{M}^{p}, f, w_{1}, w_{2}, \mathbf{n}_{1}, \mathbf{n}_{2}, \mathbf{n}_{3}\right\}
$$

where $\varepsilon_{M}^{p}$ characterizes the hardening of the matrix through equation (2), $f$ is the volume fraction of the voids (porosity), $w_{1}=a_{3} / a_{1}, w_{2}=a_{3} / a_{2}$ are two aspect ratios characterizing the shape of the voids and distribution $\left(a_{1}, a_{2}\right.$ and $a_{3}$ are respectively the lengths of the three semi-axes of the ellipsoid), and $\mathbf{n}_{1}, \mathbf{n}_{2}$ and $\mathbf{n}_{3}$ are unit vectors along the three principal directions of the ellipsoid. It is remarked here that, among the above-defined internal variables (3), $\varepsilon_{M}^{p}$ describes a property of matrix phase, while the others $\left(f, w_{1}, w_{2}, \mathbf{n}_{1}, \mathbf{n}_{2}\right.$, $\mathbf{n}_{3}$ ) characterize the microstructure of the porous metal. In addition, under the assumption that the ellipsoid characterizing the shape and orientation of the voids coincides with that of the distribution, the porous metal exhibits orthotropic behavior, with the axes of orthotropy coinciding with the principal directions of the voids. Note that these axes generally rotate with the deformation. 


\subsection{Macroscopic constitutive behavior}

The macroscopic constitutive relation of the porous material is given by the iterated variational homogenization (IVH) estimate of Agoras and Ponte Castañeda (2013), which makes use of the effective properties of an appropriately chosen "linear comparison composite" (LCC), to estimate the effective behavior of the nonlinear porous material of interest. The key idea of the IVH is to construct the porous microstructure iteratively in a self-similar fashion, such that a more accurate description of the local fields in the porous metal can be achieved through appropriate statistics (i.e., second moments). In addition, the effective behavior of the LCC is computed at each level of deformation by means of the estimates of Ponte Castañeda and Willis (1995) (to be referred to here as PCW estimates). As detailed in Agoras and Ponte Castañeda (2014), the effective yield surface of the porous material is given by

$$
\widetilde{\Phi}^{I V H}(\overline{\boldsymbol{\sigma}} ; \mathbf{s})=\left(\overline{\bar{\sigma}}_{e}^{(1)}(\overline{\boldsymbol{\sigma}} ; \mathbf{s})\right)^{2}-\sigma_{y}^{2}=0
$$

where $\widetilde{\Phi}^{I V H}$ is the effective yield function, and $\overline{\bar{\sigma}}_{e}^{(1)}$ denotes the square root of the second moment of the equivalent stress field over the matrix phase, depending on both the macroscopic stress $\overline{\boldsymbol{\sigma}}$ and internal variables s. For given $\overline{\boldsymbol{\sigma}}$ and s, the determination of $\overline{\bar{\sigma}}_{e}^{(1)}$ in $(4)$ requires the solution of a system of $N$ nonlinear equations

$$
\overline{\bar{\sigma}}_{e}^{(1)}=\left[\mathcal{F}_{[i]}\left(r_{[2]}, \ldots, r_{[N]}\right)\right]^{1 / 2}, \quad i=1, \ldots, N
$$

where the functions $\mathcal{F}_{[i]}\left(r_{[2]}, \ldots, r_{[N]}\right)$ are defined by expressions $(29)$ in the Appendix, the variables $r_{[i]}>0 \quad(i=2, . ., N)$ correspond to the remaining $N-1$ unknowns (see Agoras and Ponte Castañeda, 2014 for their physical meaning), and $N$ is the number of iterations used in IVH. It should be mentioned here that the accuracy of the IVH improves progressively with increasing $N$. However, the fast convergence of the IVH with increasing values of $N$ (Agoras and Ponte Castañeda, 2013) allows the use of relatively small numbers of iterations $N$ to get accurate results, and hence, $N=10$ is used in this work, which can be shown to give sufficient accurate predictions.

In particular, when porous materials consisting of initially spherical voids distributed isotropically in the matrix $\left(w_{1}=w_{2}=1\right)$ are subjected to purely hydrostatic loading condition $\overline{\boldsymbol{\sigma}}=\bar{\sigma}_{m} \mathbf{I}$, where $\bar{\sigma}_{m}=\operatorname{tr} \overline{\boldsymbol{\sigma}} / 3$ and $\mathbf{I}$ is the second-order identity tensor, the solution to 
the system of equations (5) can be shown to be given by

$$
\overline{\bar{\sigma}}_{e}^{(1)}=\frac{3}{2}\left(\sum_{j=1}^{N} \frac{1-c_{[j]}^{(2)}}{\sqrt{c_{[j]}^{(2)}}}\right)^{-1}\left|\bar{\sigma}_{m}\right|, \quad r_{[i]}=\left(\sqrt{\frac{c_{[i]}^{(2)}}{c_{[1]}^{(2)}} \prod_{k=1}^{i-1} c_{[k]}^{(2)}}\right)^{-1}, \quad i=2, \ldots, N .
$$

where the $c_{[i]}^{(2)}>0 \quad(i=1, \ldots, N)$ are incremental volume fractions in the IVH model, which are required to satisfy the condition $f=\prod_{i=1}^{N} c_{[i]}^{(2)}$. It should be noted here that the specific values of $c_{[i]}^{(2)} \quad(i=1, \ldots, N)$ will not significantly affect the accuracy of the IVH (Agoras and Ponte Castañeda, 2014) and they can be simply chosen as $c_{[i]}^{(2)}=f^{1 / N} \quad(i=1, \ldots, N)$. For general microstructures and applied loadings, the system of equations (5) has to be solved numerically by means of an appropriate method, e.g., the Newton-Raphson method, where the above solution (6) can be used as an initial guess.

The macroscopic constitutive behavior of the porous metal is governed by the normality rule:

$$
\overline{\mathbf{D}}=\dot{\lambda} \mathbf{N}, \quad \mathbf{N} \equiv \frac{\partial \widetilde{\Phi}^{I V H}}{\partial \overline{\boldsymbol{\sigma}}}=2 \overline{\bar{\sigma}}_{e}^{(1)} \frac{\partial \overline{\bar{\sigma}}_{e}^{(1)}}{\partial \overline{\boldsymbol{\sigma}}},
$$

where $\overline{\mathbf{D}}$ is the macroscopic Eulerian strain-rate tensor, $\dot{\lambda} \geq 0$ is the plastic multiplier, which can be determined from the "consistency condition," as will be discussed in section 2.4, and $\mathbf{N}$ is the normal to the yield surface. For the effective yield function $\widetilde{\Phi}$ defined by relation (4) and (5), it can be shown (Agoras and Ponte Castañeda, 2014) that

$$
\mathbf{N}=\frac{3}{\gamma} \widehat{\mathbb{M}}_{[N]}^{P C W}\left(r_{[j]}\right) \overline{\boldsymbol{\sigma}}, \quad \text { with } \quad \gamma \equiv f \frac{1-c_{[1]}^{(2)}}{c_{[1]}^{(2)}}+\sum_{i=2}^{N}\left(1-c_{[i]}^{(2)}\right)\left(\prod_{j=i+1}^{N} c_{[j]}^{(2)}\right) \frac{1}{r_{[i]}},
$$

where $\widehat{\mathbb{M}}_{[N]}^{P C W}\left(r_{[j]}\right)$ is the normalized viscous compliance tensor given by expression (28) in the Appendix.

\subsection{Evolution of the internal variables}

When the porous metals undergo finite-strain deformation, the internal variables defined in (3) evolve, and in turn, affect the instantaneous effective constitutive relation of the materials. In this subsection, evolution equations for the internal variables defined in (3), characterizing both the hardening of matrix and the microstructure of the porous metals, are developed. 
For the evolution of the accumulated plastic strain $\varepsilon_{M}^{p}$, following Gurson (1977), we make use of the condition that the macroscopic plastic work, $\overline{\boldsymbol{\sigma}} \cdot \overline{\mathbf{D}}=\dot{\lambda} \overline{\boldsymbol{\sigma}} \cdot \mathbf{N}$, is equal to the microscopic plastic work in the matrix phase, $(1-f) \sigma_{y} \dot{\varepsilon}_{M}^{p}$, such that

$$
\dot{\varepsilon}_{M}^{p}=\dot{\lambda} \frac{\overline{\boldsymbol{\sigma}} \cdot \mathbf{N}}{(1-f) \sigma_{y}},
$$

It is recalled in this context that the tensile yield stress $\sigma_{y}$ depends on the accumulated plastic strain $\varepsilon_{M}^{p}$ through relation (2). It is also remarked that it should be possible, at least in principle, to make use of the homogenization method to estimate the overall hardening. However, given that the main focus of the present work is on the microstructure evolution, the use of more sophisticated evolution schemes for the hardening will not be pursued here.

Considering the incompressibility of the matrix phase, the change in volume of the porous material equals the change in volume of the voids, and the evolution law for the porosity $f$ takes the form (Gurson, 1977)

$$
\dot{f}=(1-f) \bar{D}_{k k}
$$

where it is recalled that $\overline{\mathbf{D}}$ is the macroscopic Eulerian strain-rate tensor. Note that this expression can also be consistently derived from the variational homogenization methods (Ponte Castañeda and Zaidman, 1994).

The evolution of the shape and orientation of the voids (and distribution) depends on the local fields in a very complicated fashion, which is in practice, extremely difficult to determine exactly. However, for the purpose of homogenization, it is sufficient to know how the average shape and orientation of the voids (and distribution) evolve. Following the work of Ponte Castañeda and Zaidman (1994), Kailasam and Ponte Castañeda (1997, 1998) and Aravas and Ponte Castañeda (2004), it is assumed that the shape of the voids and distribution remain ellipsoidal during the deformation process, but change their aspect ratios and orientations. More specifically, it is assumed that, on the average, the voids (and distribution) change their shape and orientation with the average strain-rate and spin field in the voids. Making use of these assumptions and of standard kinematical arguments for evolution of ellipsoid under a uniform field, it follows that the evolution law of the aspect ratios $w_{\alpha} \quad(\alpha=1,2)$ is given by

$$
\dot{w}_{\alpha}=w_{\alpha}\left(\mathbf{n}_{3} \otimes \mathbf{n}_{3}-\mathbf{n}_{\alpha} \otimes \mathbf{n}_{\alpha}\right) \cdot \overline{\mathbf{D}}^{(2)}, \quad \alpha=1,2,
$$


where $\overline{\mathbf{D}}^{(2)}=\mathbb{A}^{(2)} \overline{\mathbf{D}}$ is the average strain-rate tensor in the void phase, $\mathbb{A}^{(2)}$ is the strain-rate concentration tensor determined by expressions (31) and (32) in the Appendix.

Correspondingly, the following evolution equations are obtained for the unit vectors $\mathbf{n}_{\alpha}$ $(i=1,2,3)$ along the three principal directions of the voids, namely

$$
\dot{\mathbf{n}}_{\alpha}=\overline{\boldsymbol{\omega}} \mathbf{n}_{\alpha}, \quad \alpha=1,2,3,
$$

where $\overline{\boldsymbol{\omega}}$ is an anti-symmetric tensor. Since the principal directions of the voids coincide with the Eulerian axes of the average deformation gradient of the voids, $\overline{\boldsymbol{\omega}}$ is determined by the well-known kinematical relation (e.g., Ogden, 1984)

$$
\overline{\boldsymbol{\omega}}=\overline{\mathbf{W}}^{(2)}+\frac{1}{2} \sum_{\substack{\alpha, \beta=1 \\ \alpha \neq \beta \\ w_{\alpha} \neq w_{\beta}}}^{3} \frac{w_{\alpha}^{2}+w_{\beta}^{2}}{w_{\alpha}^{2}-w_{\beta}^{2}}\left[\left(\mathbf{n}_{\alpha} \otimes \mathbf{n}_{\beta}+\mathbf{n}_{\beta} \otimes \mathbf{n}_{\alpha}\right) \cdot \overline{\mathbf{D}}^{(2)}\right] \mathbf{n}_{\alpha} \otimes \mathbf{n}_{\beta},
$$

where $w_{3}=a_{3} / a_{3}=1$, and $\overline{\mathbf{W}}^{(2)}$ is the average spin tensor of the voids. The tensor $\overline{\mathbf{W}}^{(2)}$ can be determined consistently from the IVH procedure in terms of the macroscopic strain-rate $\overline{\mathbf{D}}$ and macroscopic spin $\overline{\mathbf{W}}$, by means of expression (33), along with (34) and (35), as shown in the Appendix. For cases in which at least two aspect ratios are identical, special care needs to be taken, and this will be discussed later in this section.

For later use, we also provide the expression for the Jaumann derivative $\stackrel{\nabla}{\mathbf{n}_{\alpha}}$ of the orientation vectors, namely

$$
\stackrel{\nabla}{\mathbf{n}_{\alpha}}=\dot{\mathbf{n}}_{\alpha}-\overline{\mathbf{W}} \mathbf{n}_{\alpha}=(\overline{\boldsymbol{\omega}}-\overline{\mathbf{W}}) \mathbf{n}_{\alpha}, \quad \alpha=1,2,3
$$

The above equation may be rewritten in terms of the plastic spin $\overline{\mathbf{W}}^{p}=\overline{\mathbf{W}}-\overline{\boldsymbol{\omega}}$ (Dafalias, 1985; Aravas and Ponte Castañeda , 2004), representing the spin of the continuum relative to the microstructure, in the form

$$
\stackrel{\nabla}{\mathbf{n}}_{\alpha}=-\overline{\mathbf{W}}^{p} \mathbf{n}_{\alpha}, \quad \alpha=1,2,3
$$

Making use of expression (13) and (33), the plastic spin $\overline{\mathbf{W}}^{p}$ can be given explicitly by expression (36) in the Appendix.

It should be noted that when at least two of the aspect ratios are the same, certain components of $\overline{\mathbf{W}}^{p}$ become indeterminate. For example, when $w_{1}=w_{3}$, the porous metal 
becomes transversely isotropic about the $\mathbf{n}_{2}$ direction, which leaves $\bar{W}_{13}^{p}$ indeterminate. Since $\bar{W}_{13}^{p}$ then becomes inconsequential, it can be set equal to zero. In other words, $\bar{\omega}_{13}=\bar{W}_{13}$ in this case. In addition, for spherical voids where $w_{1}=w_{2}=w_{3}$, the porous metal becomes isotropic, and similarly, we can set $\overline{\mathbf{W}}^{p}=\mathbf{0}$ and $\overline{\boldsymbol{\omega}}=\overline{\mathbf{W}}$.

With the evolution laws for the internal variables provided above, the porous medium remains orthotropic throughout the deformation process, although the axes of orthotropy can rotate in general. However, it should be recalled here that separate evolution laws for the shape and orientation of the voids and distribution can be considered (Kailasam et al., 1997; Kailasam, 1998). However, this will not be pursued in this work, since the effect of distribution is not expected to be significant for porous materials at low to moderate porosities.

\subsection{The consistency condition and macroscopic hardening rate}

The consistency condition is given by

$$
\begin{aligned}
\dot{\widetilde{\Phi}}^{I V H}(\overline{\boldsymbol{\sigma}} ; \mathbf{s})= & \frac{\partial \widetilde{\Phi}^{I V H}}{\partial \overline{\boldsymbol{\sigma}}} \cdot \frac{\nabla}{\boldsymbol{\sigma}}+\frac{\partial \widetilde{\Phi}^{I V H}}{\partial \varepsilon_{M}^{p}} \dot{\varepsilon}_{M}^{p}+\frac{\partial \widetilde{\Phi}^{I V H}}{\partial f} \dot{f}+\sum_{\alpha=1}^{2} \frac{\partial \widetilde{\Phi}^{I V H}}{\partial w_{\alpha}} \dot{w}_{\alpha}+ \\
& \sum_{\alpha=1}^{3} \frac{\partial \widetilde{\Phi}^{I V H}}{\partial \mathbf{n}_{\alpha}} \cdot \stackrel{\nabla}{\alpha}_{\alpha}=0
\end{aligned}
$$

where $\frac{\nabla}{\boldsymbol{\sigma}}=\dot{\bar{\sigma}}-\overline{\mathbf{W}} \overline{\boldsymbol{\sigma}}+\overline{\boldsymbol{\sigma}} \overline{\mathbf{W}}$ is the Jaumann derivative of the macroscopic stress tensor. In connection with (16), we recall that $\dot{\varepsilon}_{M}^{p}, \dot{f}, \dot{w}_{\alpha}$ and $\stackrel{\nabla}{\mathbf{n}}_{\alpha}$ are given by the associated evolution laws (9), (10), (11) and (15) respectively. Making use of these evolution laws, the consistency condition (16) may be rewritten as

$$
\mathbf{N} \cdot \frac{\nabla}{\boldsymbol{\sigma}}-\dot{\lambda} H_{J}=0
$$

where $\dot{\lambda}$ is the plastic multiplier and $H_{J}$ is the macroscopic Jaumann hardening rate of the porous metal, given by

$$
H_{J}=H_{\varepsilon}+H_{f}+H_{w}+H_{\mathbf{n}}
$$


with

$$
\begin{aligned}
& H_{\varepsilon}=\frac{2 \overline{\boldsymbol{\sigma}} \cdot \mathbf{N}}{1-f} \frac{d \sigma_{y}}{d \varepsilon_{M}^{p}}, \quad H_{f}=-(1-f) N_{k k} \frac{\partial \widetilde{\Phi}^{I V H}}{\partial f} \\
& H_{w}=-\sum_{\alpha=1}^{2}\left[\left(\mathbf{n}_{3} \otimes \mathbf{n}_{3}-\mathbf{n}_{\alpha} \otimes \mathbf{n}_{\alpha}\right) \cdot \mathbb{A}^{(2)} \mathbf{N}\right] \frac{\partial \widetilde{\Phi}^{I V H}}{\partial w_{\alpha}} \\
& H_{\mathbf{n}}=\overline{\mathbf{\Omega}}^{p} \cdot \sum_{\alpha=1}^{3}\left(\frac{\partial \widetilde{\Phi}^{I V H}}{\partial \mathbf{n}_{\alpha}} \otimes \mathbf{n}_{\alpha}\right)
\end{aligned}
$$

where $\bar{\Omega}^{p}$ is given by expression (38) in the Appendix.

Making use of the consistency condition (17), the plastic flow rule (7) may be rewritten in standard form as

$$
\overline{\mathbf{D}}=\frac{\mathbf{N} \cdot \bar{\nabla}}{H_{J}} \mathbf{N},
$$

where it is recalled that $\mathbf{N}$ is given by (8), and $H_{J}$ is given by expressions (18) and (19).

In conclusion, the plastic flow rule (20), combined with the yield criterion (4) and evolution laws (9), (10), (11) and (15), constitute a (complete) internal variable plasticity model, referred to here, for brevity, as the IVH model. It should be emphasized that the model incorporates the effect of void rotation, which then can be applied for general loading conditions including simple shear. Under given loading conditions for the rigid-plastic porous metal, the IVH model has to be implemented incrementally, and the key ingredients of the procedure are: (1) the computation of the instantaneous response of the material at the end of each increment, and (2) the update of the internal variables (3) through the integration of the evolution laws. Here, we will adopt the backward Euler method proposed in the work of Aravas and Ponte Castañeda (2004) for the integration of the IVH model. At last, it should be emphasized that the IVH model is fully predictive, as it does not involve any fitting parameters. In other words, given the constitutive properties of the ductile matrix material and the initial values of the microstructural variables, the model provides estimates for the time-dependent, anisotropic response of the porous plastic metal.

In the next sections, we consider several applications of the IVH model. First, we provide results for the instantaneous macroscopic properties of porous materials subjected to combined shear and hydrostatic stress, with the goal of investigating the various effects of porosity, void shape and void orientation on the macroscopic yield surface of porous materi- 
als with prescribed (fixed) microstructures. Additional results for the effect of the porosity and pore shape (but not void orientation) for other types of loading conditions, as well as comparisons with other models and numerical results, have been given by Agoras and Ponte Castañeda (2013). Next, we will consider the time-dependent response of the porous material under simple shear loading, investigating the evolution of the void shape and orientation and its implications for the macroscopic response and shear localization. The results will be compared to the earlier results of Agoras and Ponte Castañeda (2014) for pure shear loading, where void rotations are not expected, in particular, to assess the effect of void rotations under simple shear conditions. Finally, we will consider more general loading conditions involving combined shear and tension under plane strain conditions to investigate the effect of stress triaxiality on the macroscopic response of the porous materials, as well as implications for shear localization.

\section{Instantaneous response: Macroscopic yield surfaces for combined shear and hydrostatic loading}

In this section, we investigate the predictions of the IVH model for the instantaneous macroscopic response of porous metals, focusing on the effect of the microstructural variables, and especially on the effect of the void rotation. For simplicity, we restrict our considerations on microstructural configurations such that the principal axis $\mathbf{n}_{2}$ of the voids is always fixed and aligned with the corresponding laboratory axis $\mathbf{e}_{2}$, so that the average void orientation is completely characterized by the angle $\theta$ formed by the microstructural vector $\mathbf{n}_{1}$ and the laboratory axis $\mathbf{e}_{1}$, as shown in Fig. 2. In this case, the microstructural variables reduce to the porosity, $f$, the two aspect ratios characterizing the ellipsoidal shape of the voids (and their distribution), $w_{1}=a_{3} / a_{1}$ and $w_{2}=a_{3} / a_{2}$, as well as the average orientation angle of the voids, $\theta$.

We examine the effect of the microstructural variables on the yield surface of a porous metal for loading conditions of the type

$$
\overline{\boldsymbol{\sigma}}=\bar{\sigma}_{m} \mathbf{I}+\bar{\sigma}_{13}\left(\mathbf{e}_{1} \otimes \mathbf{e}_{3}+\mathbf{e}_{3} \otimes \mathbf{e}_{1}\right)
$$

combining hydrostatic stress $\bar{\sigma}_{m}$ and shear stress $\bar{\sigma}_{13}= \pm \bar{\sigma}_{e} / \sqrt{3}$ (see also Fig. 2), where $\bar{\sigma}_{e}$ 


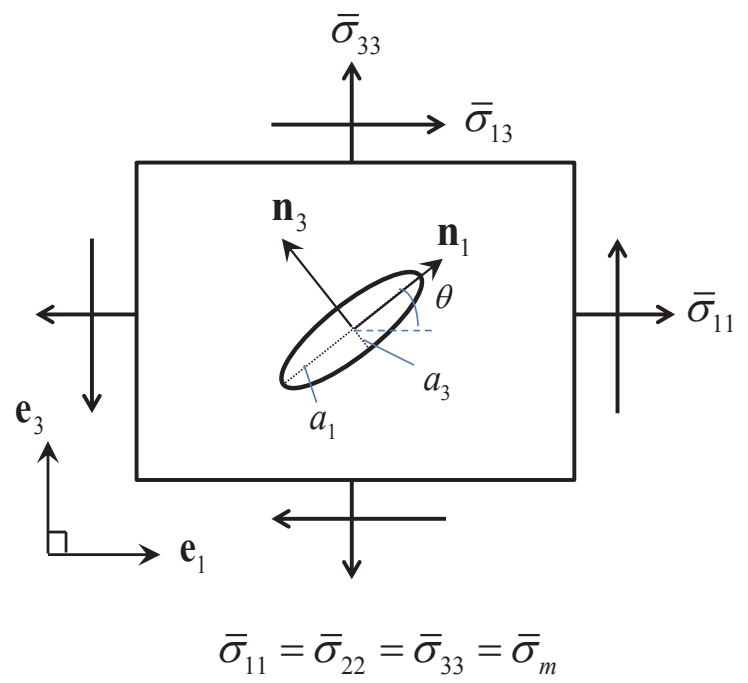

Figure 2: Schematic representation (on the plane $\mathbf{e}_{1}-\mathbf{e}_{3}$ ) of the stress states and the associated pore average shape and orientation considered in the results of Fig. 3.

is the macroscopic equivalent stress.

Figure 3(a) shows $\bar{\sigma}_{m}-\bar{\sigma}_{13}$ cross sections of the yield surfaces for $f=1 \%, 3 \%$ and $10 \%$ porosity, with the pore average shape taken in all cases to be ellipsoidal with aspect ratios $w_{1}=0.1$ and $w_{2}=0.2$, and orientation angle $\theta=0$. The main observation from Fig. 3(a) is that increasing the porosity $f$ leads to the reduction of the yield surface (especially at high stress triaxialities) and, thus, to softer behavior, as expected. In addition, note that the yield surfaces tend to become flat for low triaxialities (near the shear stress axis), especially for low porosities. This strongly nonlinear behavior is a well-known feature of porous plasticity.

On the other hand, Fig. 3(b) shows $\bar{\sigma}_{m}-\bar{\sigma}_{13}$ cross sections of the yield surfaces of materials with fixed porosity $f=10 \%$ and orientation angle $\theta=0$ for three different types of pore shape: an oblate pore shape with aspect ratios $w_{1}=w_{2}=0.2$, a prolate pore shape with $w_{1}=w_{2}=5$, and a more general, ellipsoidal pore shape with $w_{1}=0.2$ and $w_{2}=5$. In general, we observe from Fig. 3(b) that changing the pore shape (i.e., changing the aspect ratios $w_{1}$ and $w_{2}$ ) has the effect of changing both the size and the shape of the yield surface, although not its orientation, which in turn implies that the pore shape may have anisotropic hardening or softening effects. In particular, we observe that the material with oblate pores $\left(w_{1}=w_{2}=0.2\right)$ is softer than that with prolate pores $\left(w_{1}=w_{2}=5\right)$ for all combinations 
of shear $\bar{\sigma}_{13}$ and hydrostatic stress $\bar{\sigma}_{m}$. Note that the differences between these two yield curves are of the same order as those between the yield curves for porosities $f=1 \%$ and $f=3 \%$, or for porosities $f=3 \%$ and $f=10 \%$ in Fig. 3(a), indicating that the effect of the pore shape may be accordingly of the same order as that of the porosity and, therefore, quite significant. The material with more general, ellipsoidal pores $\left(w_{1}=0.2\right.$ and $\left.w_{2}=5\right)$ is softer than the other two under hydrostatic loading $\bar{\sigma}_{m}$ and harder than the other two under pure shear loading $\bar{\sigma}_{13}$, suggesting that the void shape change may have a strong hardening or a strong softening effect, depending on the loading direction.

Finally, Figs. 3(c) and 3(d) examine the effect of the pore orientation angle $\theta$ on the $\bar{\sigma}_{m}-\bar{\sigma}_{13}$ cross section of the yield surface of a porous metal with $f=10 \%$ porosity. More specifically, Fig. 3(c) compares yield curves for orientation angles $\theta=0, \pi / 4$ and $\pi / 6$ of ellipsoidal pores with aspect ratios $w_{1}=0.1$ and $w_{2}=0.2$, while Fig. $3(\mathrm{~d})$ compares yield curves for orientation angles $\theta=0, \pi / 4$ and $-\pi / 4$ of oblate pores with aspect ratios $w_{1}=w_{2}=0.2$. In the context of these results, it should be remarked that the yield stress of the material under purely hydrostatic loading $\bar{\sigma}_{m}$ is independent of the angle $\theta$, as a result of the isotropic character of this loading. Note that the symmetry of the yield curves for $\theta=\pi / 4$ and $\theta=-\pi / 4$ about the axes $\bar{\sigma}_{m}=0$ and $\bar{\sigma}_{13}=0$ (see Fig. 3(d)) reflects the corresponding symmetry of the applied loading with respect to the orientations $\theta=\pi / 4$ and $\theta=-\pi / 4$. In addition, it is observed that changes in the orientation of the pores result in changes not only in the size and shape of the yield surface, as in Figs. 3(a) and 3(b), but also in its orientation. The rotation of the yield surface induced by the corresponding rotation of the voids, as observed in Figs. 3(c) and 3(d), has important implications on the plastic anisotropy, as well as on the overall hardening/softening of the material. Note, for instance, that when the porous material is subjected to pure hydrostatic tension (or compression) $\bar{\sigma}_{m}$ the direction of the induced plastic flow, as defined by the normal $\mathbf{N}$ to the yield curves in Figs. 3(c) and 3(d), may vary substantially, depending on the orientation of the voids, e.g., while for $\theta=0$ the plastic flow is purely dilatational, for $\theta=\pi / 4$ the plastic flow involves both a dilatational and a substantial shear component. An analogous conclusion can be drawn for the case that a pure shear stress $\bar{\sigma}_{13}$ is applied. Furthermore, we observe that the hydrostatic stress $\bar{\sigma}_{m}$ required to produce (instantaneously) simple shear deformation of 


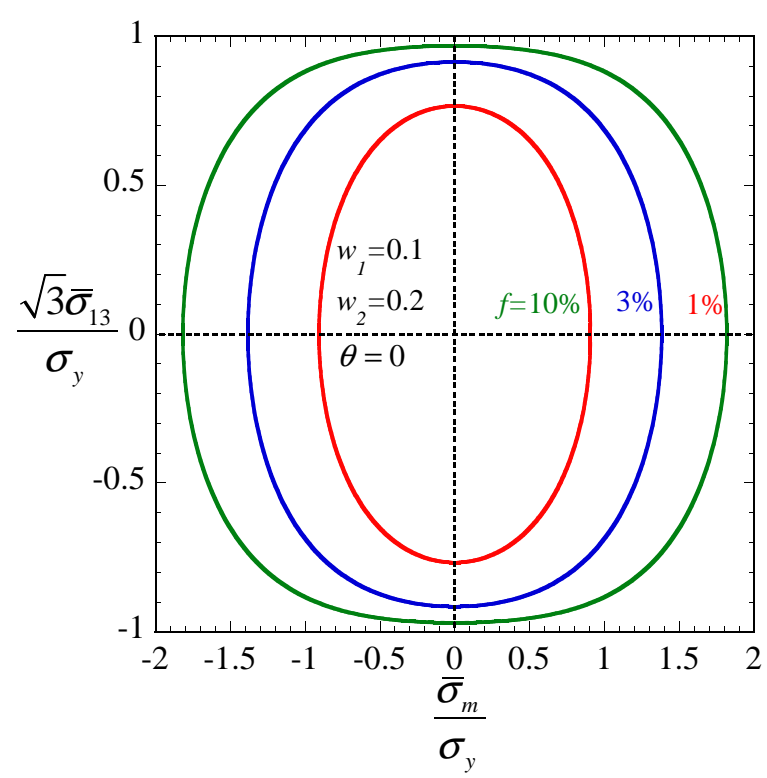

(a)

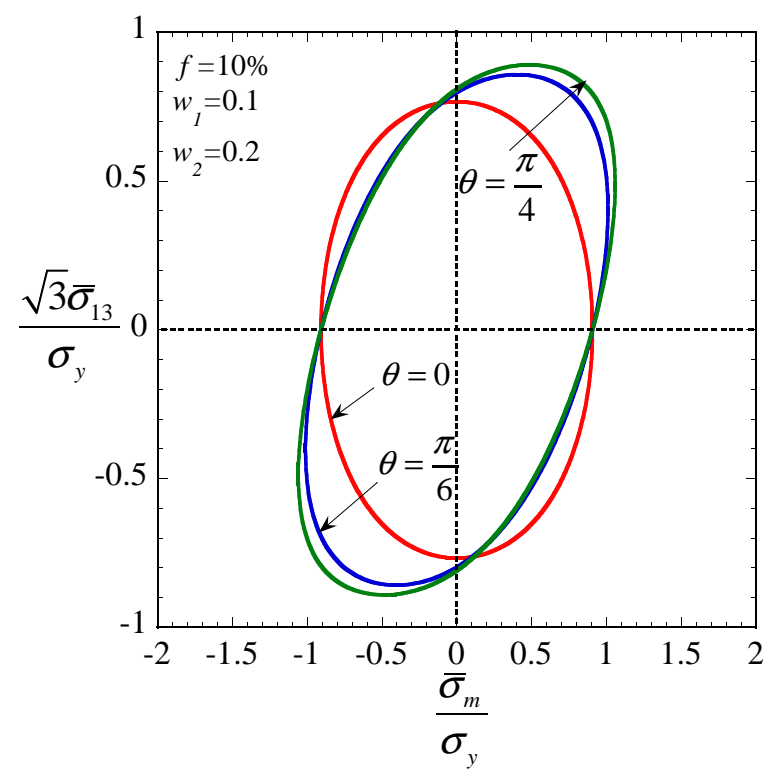

(c)

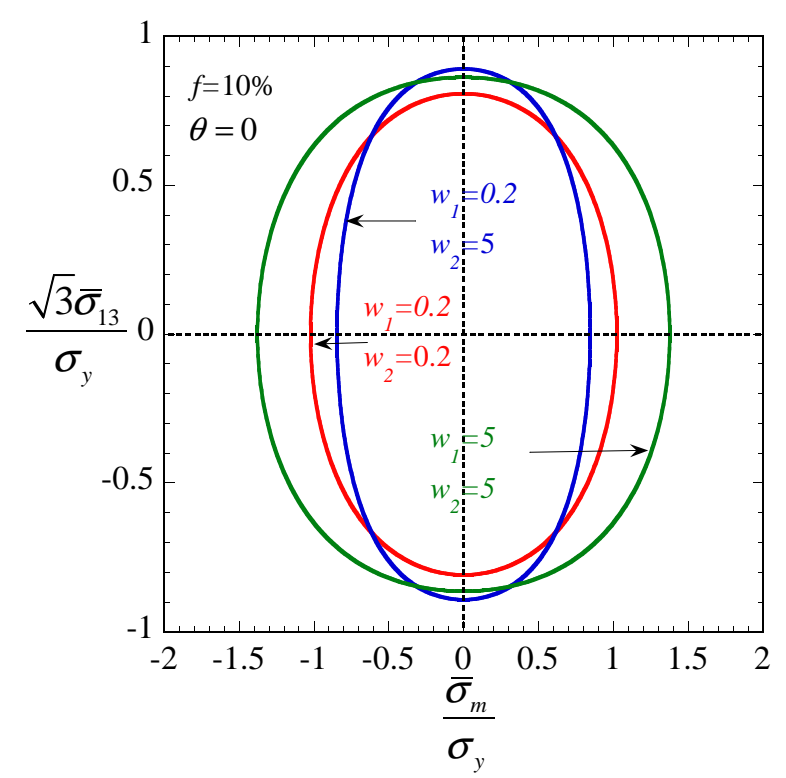

(b)

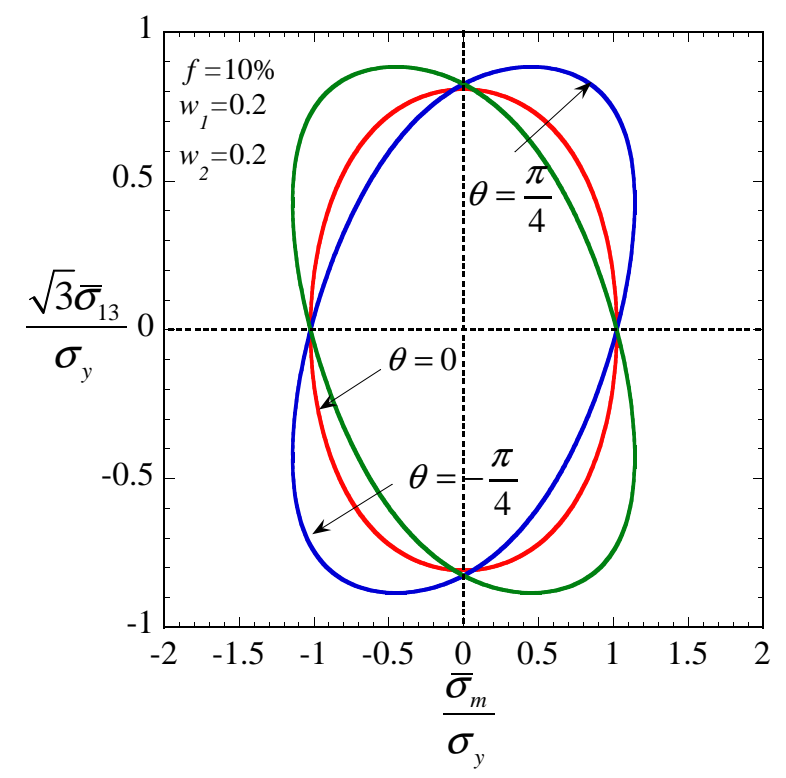

(d)

Figure 3: The effect of the (a) porosity $f$, (b) pore average shape, as defined by the aspect ratios $w_{1}$ and $w_{2},(\mathrm{c})$ and $(\mathrm{d})$ pore average orientation, as defined by the angle $\theta$ (Fig. 2), on the yield surface of a porous material subjected to a mean stress $\bar{\sigma}_{m}$ combined with a shear stress $\bar{\sigma}_{13}$ (see Fig. 2). In each case, yield curves on the plane $\bar{\sigma}_{m}-\bar{\sigma}_{13}$ are shown for different values of the relevant variables. 
the material, i.e., plastic flow parallel to the $\bar{\sigma}_{13}$ axis, is zero when the orientation of the voids is parallel to the direction of the applied shear $(\theta=0)$, but it reaches a value which is comparable to the corresponding shear stress $\bar{\sigma}_{13}$ when the voids are inclined at an angle $\theta=\pi / 4, \pi / 6$ or $-\pi / 4$ with respect to the direction of the applied shear deformation.

\section{Finite-strain response under plane strain conditions: Microstructure evolu- tion, macroscopic stress-strain behavior and localization}

In this section, we consider porous materials consisting of initially spherical voids distributed randomly and isotropically $\left(w_{1}=w_{2}=1\right)$ in a rigid-plastic matrix, with a yield stress $\sigma_{y}$ characterized by the isotropic strain hardening relation (2), where $\varepsilon_{0}=0.001$ and $M=0.1$. This material is subjected to plane strain loading conditions, prescribed by the affine velocity field $\mathbf{v}=\overline{\mathbf{L}} \mathbf{x}$, where $\overline{\mathbf{L}}$ is the velocity gradient tensor and $\mathbf{x}$ stands for the points on the boundary of the specimen. The components of $\overline{\mathbf{L}}$ are independent of $\mathbf{x}$, but not necessarily fixed in time, so that special conditions such as fixed stress triaxiality during the loading may be enforced when the components of $\overline{\mathbf{L}}$ are allowed to evolve in time. Specifically, letting $\bar{L}_{i j}$ denote the components of $\overline{\mathbf{L}}$ relative to a fixed coordinate system $\mathbf{e}_{i}$, two different cases corresponding to non-symmetric and symmetric $\overline{\mathbf{L}}$, respectively, are considered, as shown schematically in Figs. 4(a) and 4(b):

- Non-vanishing spin shear.

$$
\bar{L}=\left(\begin{array}{ccc}
0 & 0 & 1 \\
0 & 0 & 0 \\
0 & 0 & \alpha
\end{array}\right), \quad \bar{D}=\left(\begin{array}{ccc}
0 & 0 & \frac{1}{2} \\
0 & 0 & 0 \\
\frac{1}{2} & 0 & \alpha
\end{array}\right), \quad \bar{W}=\left(\begin{array}{ccc}
0 & 0 & \frac{1}{2} \\
0 & 0 & 0 \\
-\frac{1}{2} & 0 & 0
\end{array}\right), \quad \text { with } \quad \alpha \in \Re
$$

and

- Vanishing-spin shear.

$$
\bar{L}=\left(\begin{array}{ccc}
\beta & 0 & 0 \\
0 & 0 & 0 \\
0 & 0 & -1
\end{array}\right), \quad \bar{D}=\left(\begin{array}{ccc}
\beta & 0 & 0 \\
0 & 0 & 0 \\
0 & 0 & -1
\end{array}\right), \quad \bar{W}=\left(\begin{array}{lll}
0 & 0 & 0 \\
0 & 0 & 0 \\
0 & 0 & 0
\end{array}\right), \quad \text { with } \beta \geq 0
$$




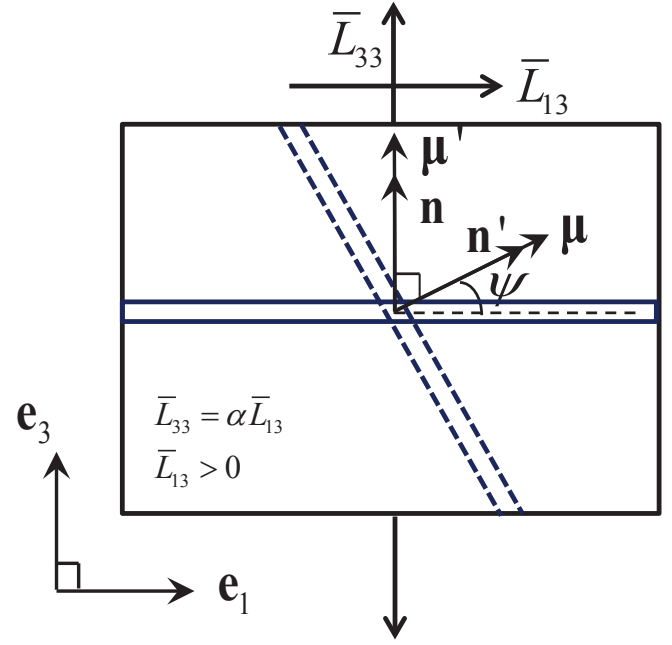

Non-vanishing spin loading

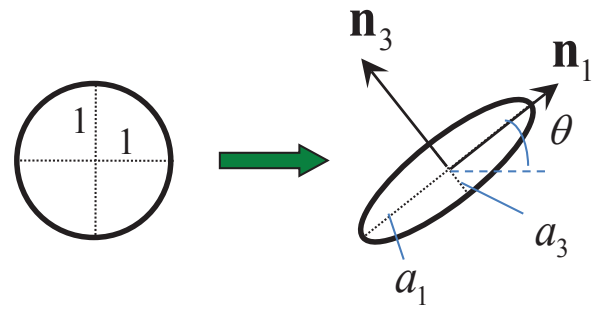

Void average deformation

(a)

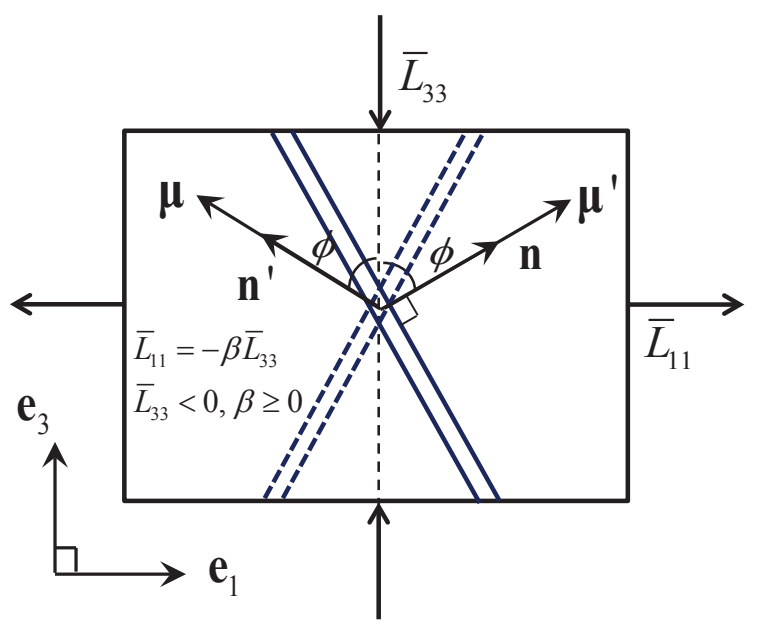

Vanishing spin loading

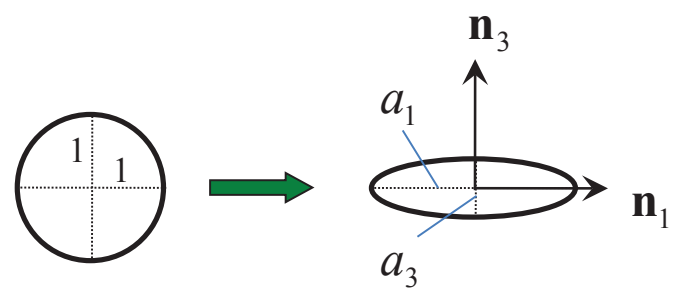

Void average deformation

Figure 4: Schematic representation of the (a) non-vanishing spin and (b) vanishing spin, plane strain loading conditions, defined by (22) and (23), respectively, along with the associated in-plane, average deformation of a representative void (sphere to ellipsoid). The void shape and orientation in the deformed state are defined by the associated aspect ratios $w_{1}=a_{3} / a_{1}$ and $w_{2}=a_{3} / a_{2}$, and the orientation angle $\theta$, respectively. The geometrical features of the localization band that may develop at a certain critical strain are also shown. 
Note that a loading of the type (22) is a combination of uniaxial straining with shear, while a loading of the type (23) corresponds to bi-axial straining. In general, a loading of the type (22) is expected to affect not only the porosity $f$ and the average void (ellipsoidal) shape, as defined by the aspect ratios $w_{1}=a_{3} / a_{1}$ and $w_{2}=a_{3} / a_{2}$, but also the average void orientation in the $\mathbf{e}_{1}-\mathbf{e}_{3}$ plane, as defined by the angle $\theta$ in Fig. 4(a). On the other hand, a loading of the type (23) affects the porosity and the average void shape, but not the void orientation, i.e., under the loading conditions (23) the void orientation vectors $\mathbf{n}_{i}$ coincide with the fixed vectors $\mathbf{e}_{i}$ throughout the deformation (see Fig. 4(b)).

For future reference, it is convenient to also introduce the macroscopic equivalent strain $\bar{E}_{e}=\int_{0}^{t} \bar{D}_{e} d t$, where $t$ denotes the time variable, and $\bar{D}_{e}=\sqrt{\frac{2}{3} \overline{\mathbf{D}}_{d} \cdot \overline{\mathbf{D}}_{d}}$ is the equivalent strain-rate (with $\overline{\mathbf{D}}_{d}$ denoting the strain-rate deviator tensor). In particular, for the case of the non-vanishing spin loading (22),

$$
\bar{D}_{e}=\frac{2}{3}\left(\bar{D}_{33}^{2}+3 \bar{D}_{13}^{2}\right)^{1 / 2}
$$

while for the case of the vanishing-spin loading (23),

$$
\bar{D}_{e}=\frac{2}{3}\left(\bar{D}_{11}^{2}-\bar{D}_{11} \bar{D}_{33}+\bar{D}_{33}^{2}\right)^{1 / 2} .
$$

As we have seen in the previous section, the variations of the various microstructural variables $\left(f, w_{1}, w_{2}\right.$ and $\left.\theta\right)$ during the deformation process may each have hardening or softening effects, the competition or synergy among which determines the overall hardening or softening of the macroscopic response of the material. As a consequence, there may be a certain critical strain during the loading process at which the softening effects may prevail over the hardening effects and, thus, the porous material may become unstable. In this work, we will consider specifically macroscopic, or material instabilities, which are characterized by localization of the plastic deformation within a thin band (or loss of strong ellipticity). As given by Rice (1977), the critical conditions for strain localization in rigid-plastic solids are:

(i) the normal $\mathbf{N}$ to the yield surface must be of the form

$$
\mathbf{N}=\frac{1}{2}(\boldsymbol{\mu} \otimes \mathbf{n}+\mathbf{n} \otimes \boldsymbol{\mu}),
$$


where $\mathbf{n}$ denotes the unit normal to the plane of the localization band and $\boldsymbol{\mu}$ is a suitably determined vector, and

(ii) the Jaumann hardening modulus $H_{J}$ must be equal to the following critical value

$$
H_{c r}=\frac{1}{2}|\boldsymbol{\mu}|^{2}\left(\bar{\sigma}_{\mu \mu}-\bar{\sigma}_{n n}\right)
$$

where $\bar{\sigma}_{\mu \mu}$ and $\bar{\sigma}_{n n}$ are the normal stresses in the directions of $\boldsymbol{\mu}$ and $\mathbf{n}$, respectively.

Note that condition (26) is equivalent to requiring the localization plane to be a nondeforming plane. It can be easily shown that under the plane strain loading conditions (22) or (23) there exist two non-deforming planes (interchange the role of $\mathbf{n}$ and $\boldsymbol{\mu}$ in (26)), both of which are parallel to the constrained direction $\mathbf{e}_{2}$. For the non-vanishing spin loading (22), one of these planes has normal $\mathbf{e}_{3}$ and the other has normal oriented at an angle $\psi=\tan ^{-1} \alpha$ with respect to $\mathbf{e}_{1}$ (see Fig. 4(a)). On the other hand, for the vanishing-spin loading (23), the two non-deforming planes are symmetrical relative to the directions $\mathbf{e}_{1}$ and $\mathbf{e}_{3}$, with their normals forming equal angles $\phi=\tan ^{-1} \sqrt{\beta}$ with the direction $\mathbf{e}_{3}$ (see Fig. 4(b)). Note also that the vector $\boldsymbol{\mu}$ may be easily obtained in terms of $\mathbf{n}$ and $\mathbf{N}$ by solving the linear equation (26), given that the normal $\mathbf{N}$ to the yield surface may be readily determined by means of the IVH estimate (8) for any given microstructural configuration and stress state during a loading of the type (22) or (23). It can also be shown that the vectors $\mathbf{n}$ and $\boldsymbol{\mu}$ are symmetrical with respect to the principal directions of $\mathbf{N}$ on the plane $\mathbf{n}-\boldsymbol{\mu}$, which in the case of the vanishing spin loading (23) coincide with the fixed directions $\mathbf{e}_{1}$ and $\mathbf{e}_{3}$. An implication of the latter result is that $\bar{\sigma}_{\mu \mu}=\bar{\sigma}_{n n}$ ( since $\overline{\boldsymbol{\sigma}}$ is co-axial with $\mathbf{N}$ ) and, therefore, the critical condition (27) reduces to $H_{c r}=0$ for the case of the vanishing-spin loading (23). Moreover, it should be mentioned that for vanishing-spin loading (23), the critical condition $H_{c r}=0$ is reached on the two possible localization planes simultaneously, due to symmetry. However, for non-vanishing spin loading (22), the critical condition (27) on the two possible localization planes are not met at the same time. In fact, for the loading (22) considered in this work, it is found that the localization condition is always first met on the plane with unit normal $\mathbf{e}_{3}$, which is parallel to the shear loading plane. For this reason, only the critical conditions corresponding to this plane are shown in the following results for non-vanishing spin loading (22). 
It should also be recalled that the predictions of the IVH model for vanishing-spin loadings (23), with $\beta$ chosen to be constant throughout any given deformation history (fixed strain trixiality) have been discussed in detail in Agoras and Ponte Castañeda (2014). In the present work, we focus on the predictions of the IVH model for the non-vanishing spin loading (22). In order to highlight the effect of void rotations, however, we will compare the new IVH results for non-vanishing spin loadings (22) with corresponding IVH results for vanishingspin loadings (23). More specifically, in subsection 4.1, we consider the special cases $\alpha=0$ in (22) and $\beta=1$ in (23), corresponding to simple and pure shear deformation, respectively. In subsection 4.2 we also consider plane strain loadings of the type (22) and (23), where the parameters $\alpha$ and $\beta$ are computed such that the stress triaxiality $X_{\sigma} \equiv \bar{\sigma}_{m} / \bar{\sigma}_{e}$ is held fixed to a prescribed value throughout the deformation. Note that, constitutive models of the Gurson type (e.g., Nahshon and Hutchinson, 2008), which account for the porosity but not for the shape and orientation of the voids, can not distinguish between the non-vanishing spin and vanishing-spin type of loadings considered in this work.

In addition, it should be remarked that for all results discussed in the following subsections the critical conditions for strain localization $\left(H_{J}=H_{c r}\right)$ are labelled with filled circles. At this point, we recall that for the non-vanishing spin loading (22), the critical condition is first reached on the plane parallel to the shear loading plane and, for this reason, only the critical conditions corresponding to this plane are marked in the following results. Although shown for completeness, it should be emphasized that any IVH results beyond the filled circles may not be physically relevant, since a post-bifurcation study (which is beyond the scope of the present work) would be needed after the onset of localization.

Finally, it should be recalled that under conditions of void collapse, i.e., when the aspect ratios $w_{1}$ and $w_{2}$ assume very small (or very large) values, the hypothesis adopted in the context of the IVH model that the pore shape remains ellipsoidal may become questionable on physical grounds, given the facts that contact of the void surfaces would be expected to take place prior to the complete void closure (Hutchinson and Tvergaard, 2012), or that hard particles could interfere with void collapse (when the voids nucleate from such hard particles). For these reasons, and given that for the loading conditions considered in this work we always have $w_{1} \leq w_{2} \leq 1$, conditions of considerably distorted pore shapes corresponding 
to the (arbitrarily chosen) aspect ratio $w_{1}=0.1$ will be marked in the plots by open squares. Therefore, any theoretical predictions beyond the open squares will have to be subjected to careful scrutiny. For example, when contact of the void surfaces is expected to take place, the onset of localization instabilities may be delayed, or even invalidated, by such contacts. Similarly, the presence of hard particles may also delay, or prevent altogether, the onset of localization instabilities, depending on the precise loading conditions. In this work, we will not attempt to directly account for the effects of contact of void faces, or of hard particles, and we will simply make use of the above-mentioned open squares to indicate that the predictions of the present model may need to be modified to account for such effects beyond the open squares. On the other hand, the predictions of the model may be directly relevant for material systems with higher initial porosities, such as those resulting from certain powder metallurgy processes after compaction and sintering.

\subsection{Simple and pure shear loading}

First of all, it should be remarked that both simple shear $(\alpha=0$ in expression (22)) and pure shear ( $\beta=1$ in expression (23)) deformations are isochoric loadings $\left(\bar{D}_{k k}=0\right)$ and, therefore, do not alter the porosity $f$ in the material. The main difference between these loadings is that, while simple shear induces the evolution of both the average void shape and orientation, pure shear produces changes in the void shape, but not in the void orientation.

Figure 5 shows plots of the predictions of the IVH model for the internal and macroscopic variables, as functions of the applied strain $\bar{E}_{e}$ under simple shear deformation, for initial porosities $f_{0}=1 \%, 3 \%, 10 \%$ and $30 \%$ ( $f=f_{0}=$ fixed). We observe that the aspect ratios $w_{1}$ (Fig. 5(a)) and $w_{2}$ (Fig. 5(b)) decrease monotonically with increasing strain $\bar{E}_{e}$ and tend to zero as $\bar{E}_{e} \rightarrow \infty$, indicating that the average void shape deforms continuously from spherical $\left(w_{1}=w_{2}=1\right)$ to flat $\left(w_{1}, w_{2} \rightarrow 0\right)$, while the average orientation of the voids (Fig. 5(c)), as defined by the angle $\theta$ (see Fig. 2), undergoes a continuous, clockwise rotation towards the horizontal axis. As expected, the yield stress of the matrix $\sigma_{y}$ (Fig. 5(d)) increases monotonically with increasing strain $\bar{E}_{e}$ with a rate that is initially very large, but then slows down as $\bar{E}_{e}$ continues to increase. Note further that the value of the porosity $f_{0}$ does not have a significant effect on the evolution of the internal variables. 


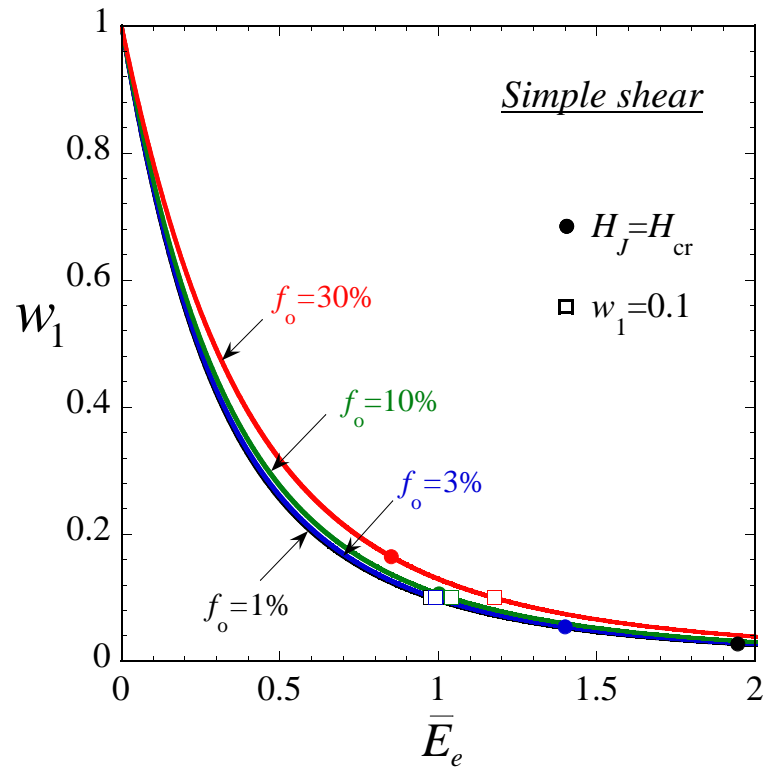

(a)

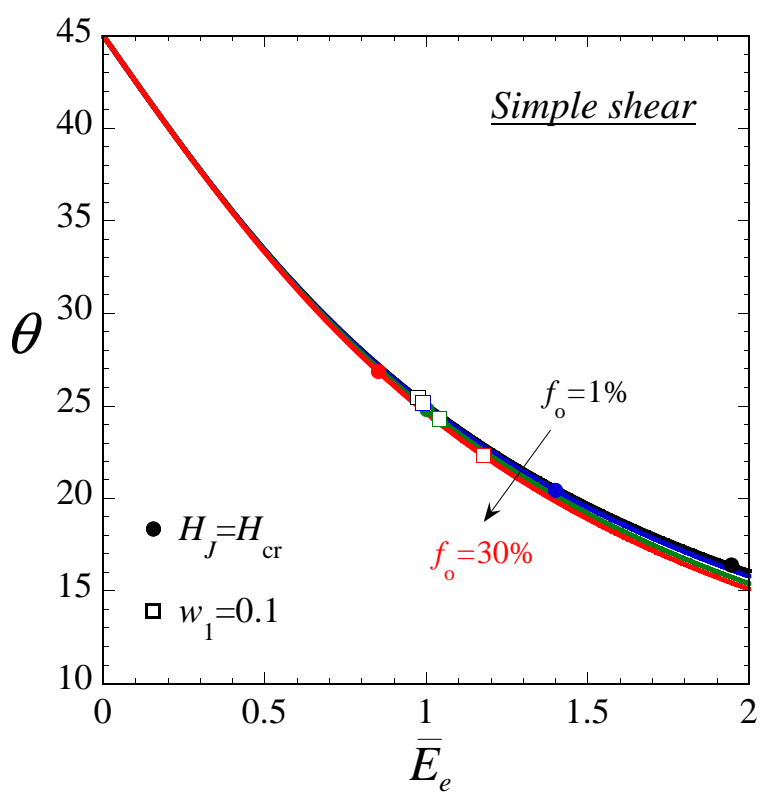

(c)

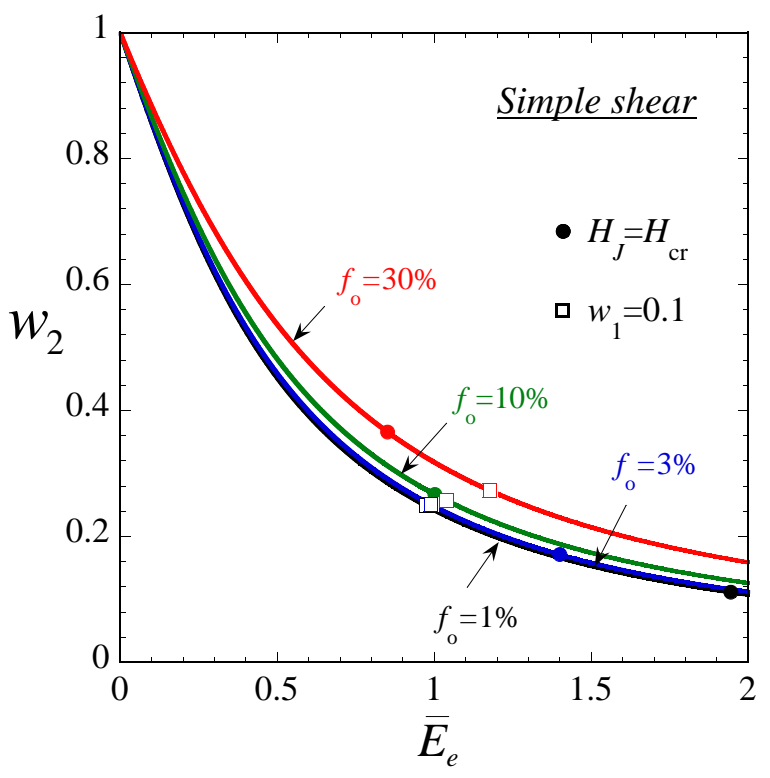

(b)

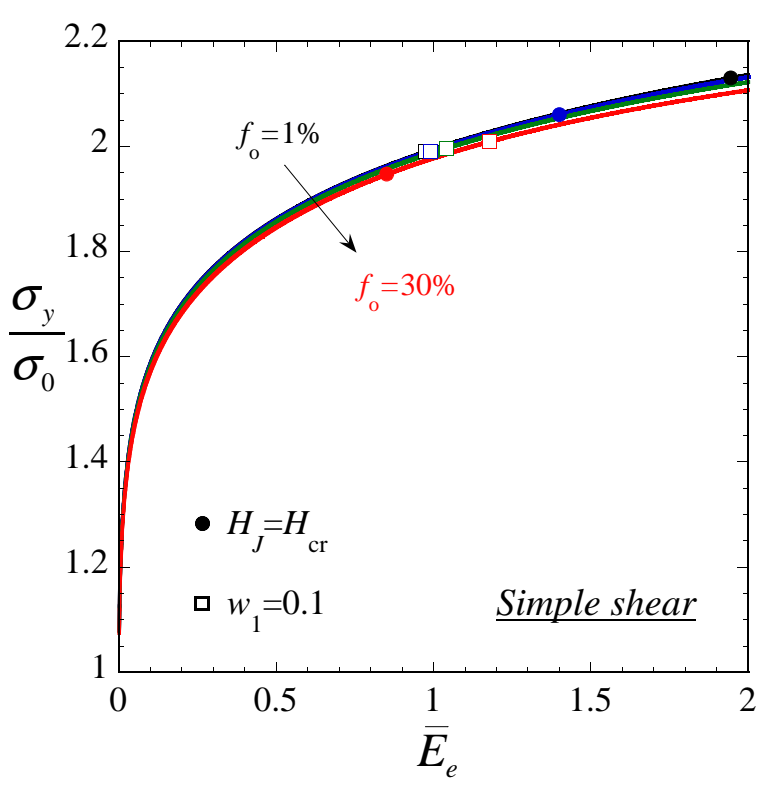

(d)

Figure 5: IVH results for simple shear deformation of initially isotropic porous materials with porosities $f=1 \%, 3 \%, 10 \%$ and $30 \%\left(f=f_{0}=\right.$ fixed $)$. Plots are shown for the evolution of the void aspect ratios (a) $w_{1}=a_{3} / a_{1}$ and (b) $w_{2}=a_{3} / a_{2}$, (c) void orientation angle $\theta$, (d) yield stress $\sigma_{y}$ of the matrix ... 


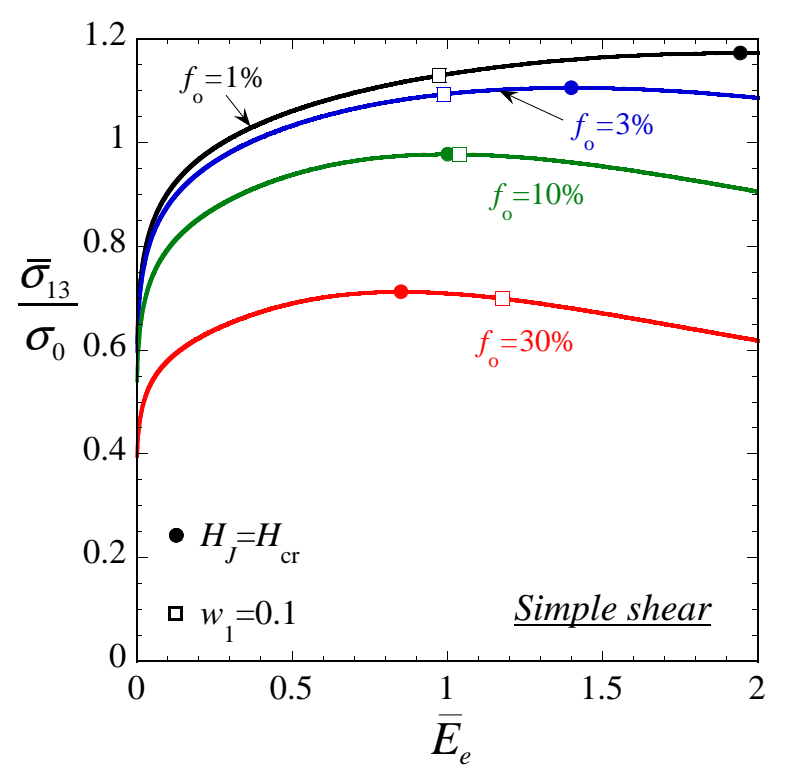

(e)

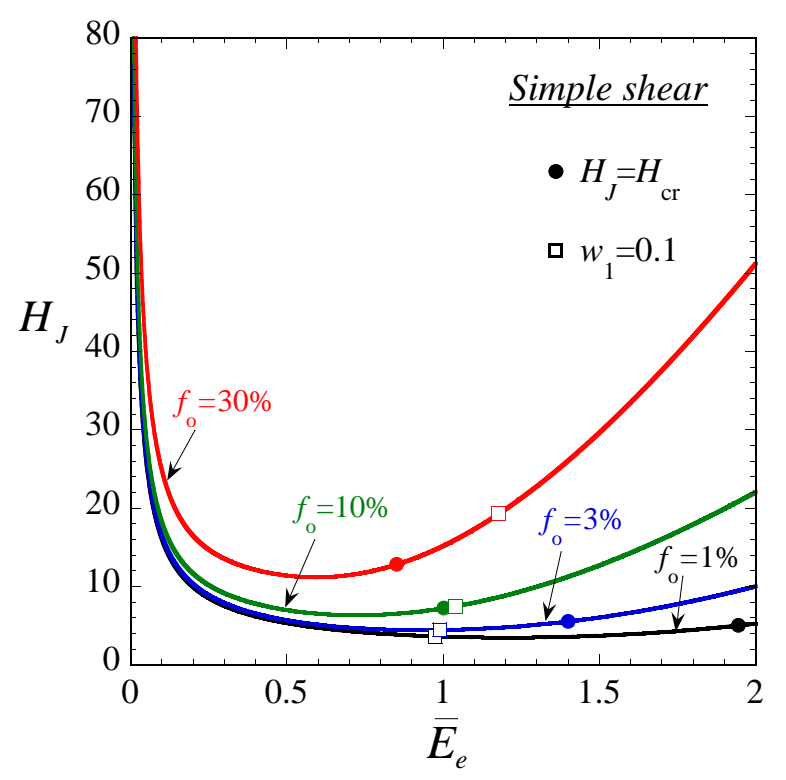

$(\mathrm{g})$

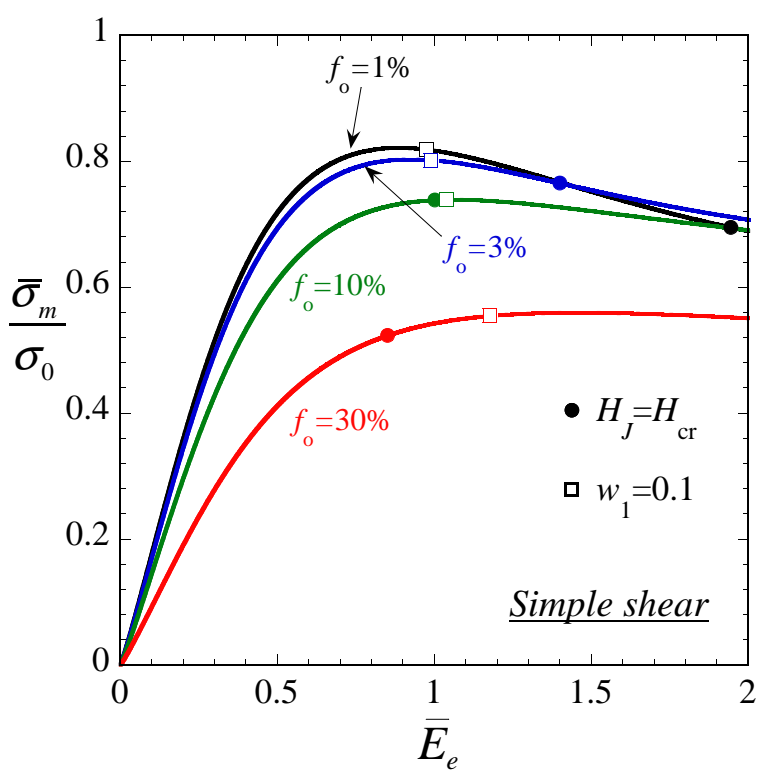

(f)

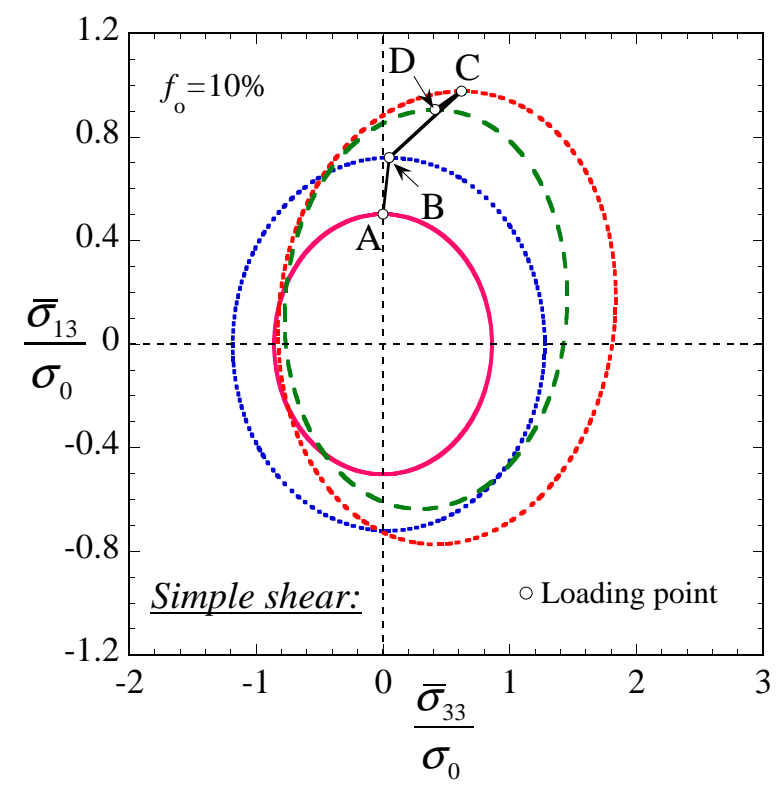

(h)

Figure 5: ... (e) average shear stress $\bar{\sigma}_{13}$, (f) average mean stress $\bar{\sigma}_{m}$ and (g) macroscopic Jaumann hardening rate $H_{J}$. (h) The actual stress state (loading point) and associated $\bar{\sigma}_{13}-\bar{\sigma}_{33}$ cross section of the yield surface for the material with $f=10 \%$ porosity are shown at the strain levels: (A) $\bar{E}_{e}=0$, (B) $\bar{E}_{e}=0.037$, (C) $\bar{E}_{e}=1.095$ and (D) $\bar{E}_{e}=1.999$; the remaining non-zero (fixed) stress components on the yield curves shown are: (A) $\bar{\sigma}_{11}=0$ and $\bar{\sigma}_{22}=0$, (B) $\bar{\sigma}_{11}=0.051 \sigma_{0}$ and $\bar{\sigma}_{22}=0.047 \sigma_{0}$, (C) $\bar{\sigma}_{11}=0.876 \sigma_{0}$ and $\bar{\sigma}_{22}=0.722 \sigma_{0}$, and (D) $\bar{\sigma}_{11}=0.981 \sigma_{0}$ and $\bar{\sigma}_{22}=0.680 \sigma_{0}$. 
From the results of Fig. 5(e) for the macroscopic shear stress $\bar{\sigma}_{13}$, we observe that the porous metal exhibits a softer behavior for larger values of the porosity $f_{0}$ (note, for example, that $\bar{\sigma}_{13}$ is substantially smaller for $f_{0}=30 \%$ than for $f_{0}=1 \%$ porosity), as expected on physical grounds. In addition, we observe that, for any given $f, \bar{\sigma}_{13}$ increases initially with increasing $\bar{E}_{e}$ up to a certain critical strain, depending on $f$, at which it reaches a maximum (marked by a filled circle), and then drops continuously with further increases in $\bar{E}_{e}$. The aforementioned critical strain corresponds to the situation where the Jaumann hardening modulus $H_{J}$ in Fig. $5(\mathrm{~g})$ reaches the critical value $H_{c r}$, implying the localization of the plastic deformation in a shear band, parallel to the shear plane of the loading (see Fig. 4(a)). The initial hardening regime in the stress-strain response of Fig. 5(e) is a consequence of the corresponding strain hardening of the matrix (Fig. 5(d)), while the subsequent softening regime is induced by the anisotropic evolution of the microstructure (Figs. 5(a)-5(c)), since the porosity $f$ does not change during the loading. From the results of Fig. 5(e), we also observe that the onset of localization occurs at smaller strains for higher values of the porosity $f$, indicating that the softening effect of the anisotropic evolution of the microstructure may be stronger for higher porosities.

The main observation from the results of Fig. $5(\mathrm{f})$ is that the average mean stress $\bar{\sigma}_{m}$ produced in the porous material by the applied simple shear deformation reaches unexpectedly large values. In particular, for any given porosity $f$, we observe that $\bar{\sigma}_{m}$ is initially zero, but increases monotonically up to a certain strain level and then drops continuously with increasing strain. Note that, for each value of $f$, the maximum value of the mean stress $\bar{\sigma}_{m}$ is comparable with the corresponding maximum shear stress $\bar{\sigma}_{13}$, although $\bar{\sigma}_{m}<\bar{\sigma}_{13}$ (see Figs. 5(e) and 5(f)).

Fig. 5(h) shows additional results for the evolution of the stress state (loading point) in the material with $f=10 \%$ porosity. In particular, this figure shows plots of the $\bar{\sigma}_{13}-\bar{\sigma}_{33}$ cross section of the yield surface, including the loading point, under the (fixed) conditions: (A) $\bar{E}_{e}=0, \bar{\sigma}_{11}=0$ and $\bar{\sigma}_{22}=0$, (B) $\bar{E}_{e}=0.037, \bar{\sigma}_{11}=0.051 \sigma_{0}$ and $\bar{\sigma}_{22}=0.047 \sigma_{0}$, (C) $\bar{E}_{e}=1.095, \bar{\sigma}_{11}=0.876 \sigma_{0}$ and $\bar{\sigma}_{22}=0.722 \sigma_{0}$, and (D) $\bar{E}_{e}=1.999, \bar{\sigma}_{11}=0.981 \sigma_{0}$ and $\bar{\sigma}_{22}=0.680 \sigma_{0}$. From these results, we observe that the yield surface expands from $\bar{E}_{e}=0$ to $\bar{E}_{e}=1.03$ and contracts from $\bar{E}_{e}=1.03$ to $\bar{E}_{e}=1.999$, reflecting accordingly the effect 


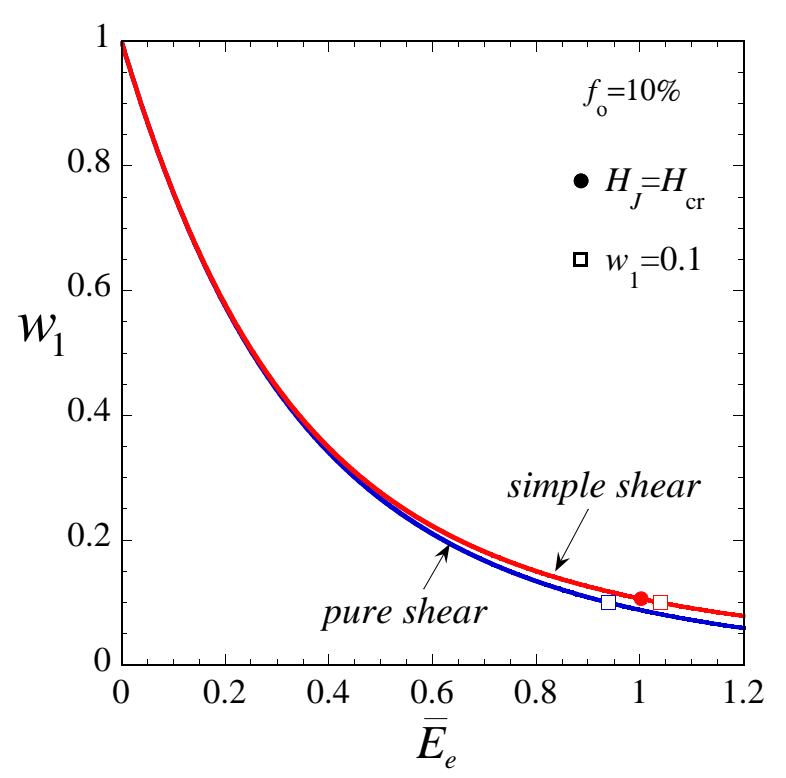

(a)

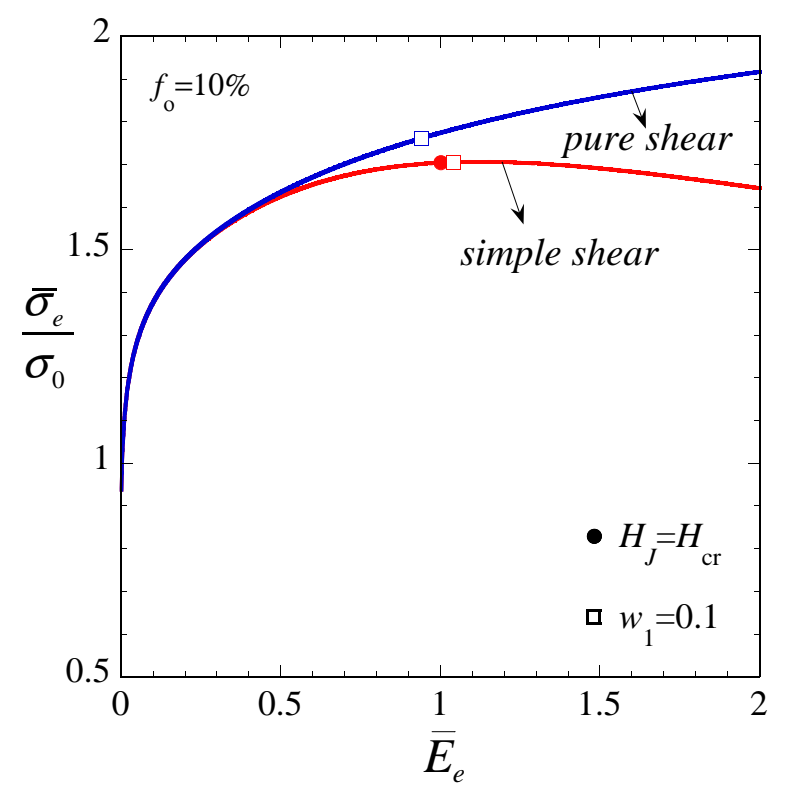

(c)

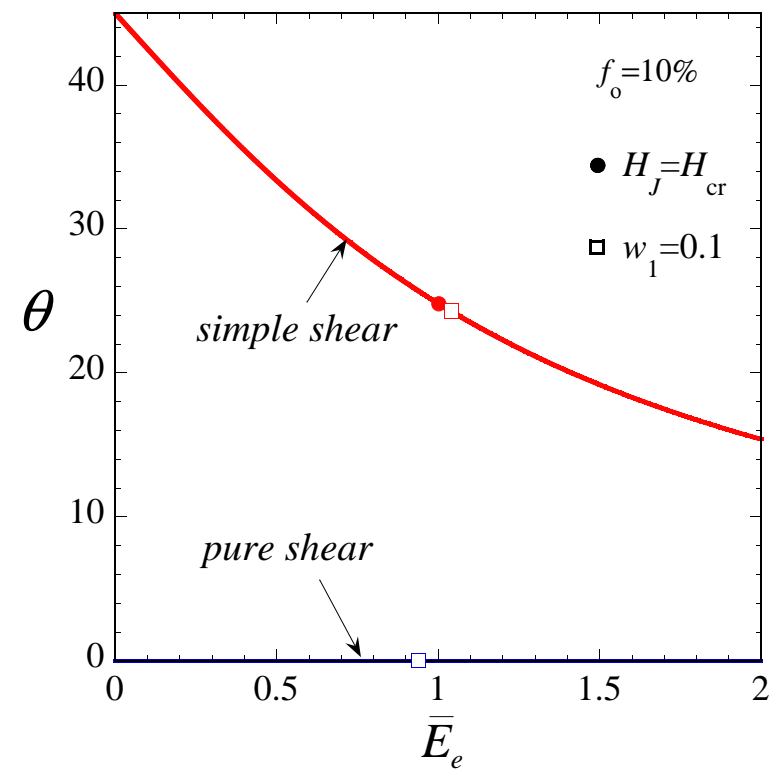

(b)

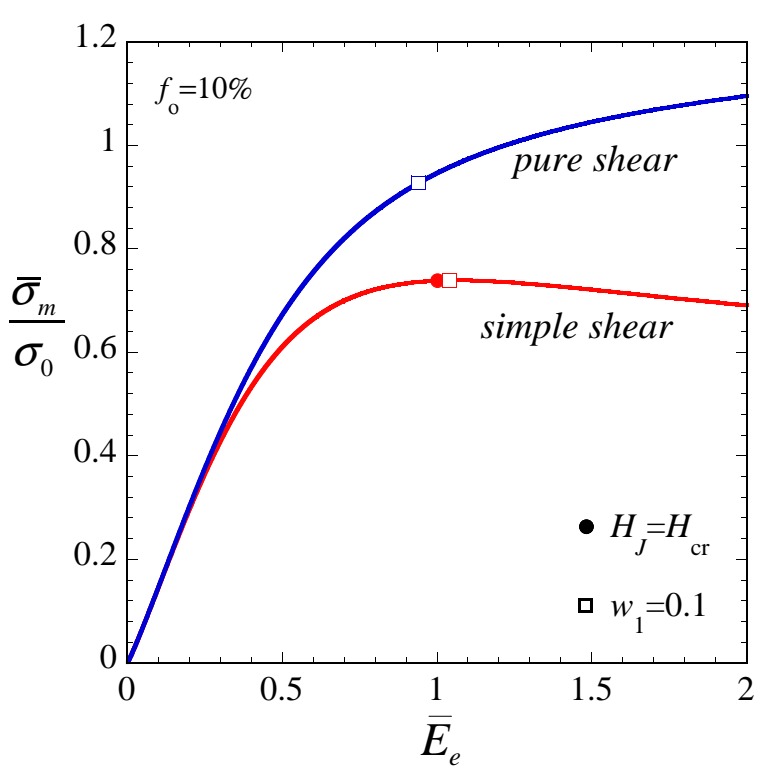

(d)

Figure 6: Comparison of IVH results for simple shear deformation of an initially isotropic porous material with $f=10 \%$ porosity with corresponding results for pure shear deformation. Plots are shown for the evolution of the (a) void aspect ratio $w_{1}$, (b) void orientation angle $\theta,(\mathrm{c})$ average equivalent stress $\bar{\sigma}_{e},(\mathrm{~d})$ average mean stress $\bar{\sigma}_{m}, \ldots$ 


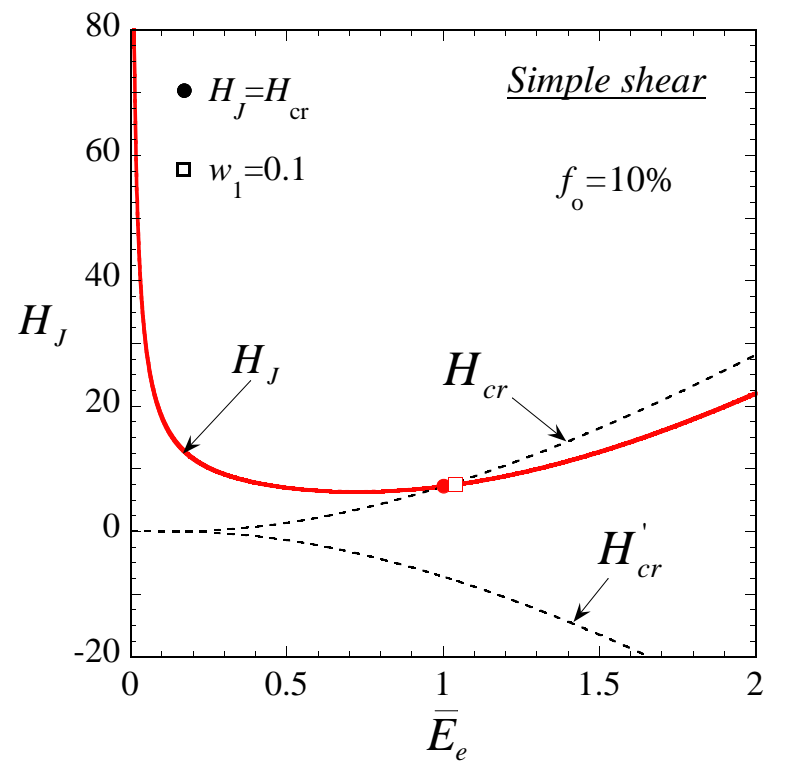

(e)

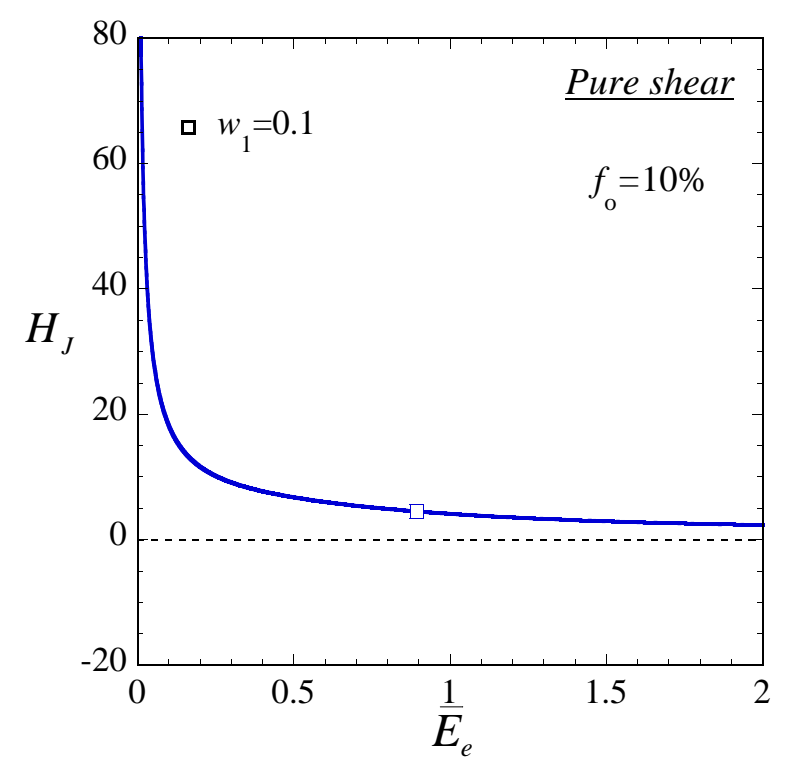

(f)

Figure 6: ... and macroscopic Jaumann hardening rate $H_{J}$ for (e) simple shear and (f) pure shear.

of the strain hardening of the matrix, as well as the hardening and softening effects of the anisotropic evolution of the microstructure (Figs. 5(a)-5(c)) on the macroscopic response of the material (Figs. 5(e) and 5(f)). In addition, we observe that the yield surface rotates and translates along both the $\bar{\sigma}_{13}$ and the $\bar{\sigma}_{33}$ axis (as well as along the axes $\bar{\sigma}_{11}$ and $\bar{\sigma}_{22}$ ) with increasing strain, which in turn leads to the gradual development of large hydrostatic stresses $\bar{\sigma}_{m}$ during the simple shear deformation of the material (notice the shift of the loading point with increasing $\bar{E}_{e}$ ), as observed in Fig. 5(f).

Figure 6 compares the response of a porous material with $f=10 \%$ porosity under simple and pure shear deformations. The results of Fig. 6(a) for $w_{1}$ show that the evolution of the pore shape under simple shear is very similar with that under pure shear, with $w_{1}$ being only slightly larger in the former than in the latter case. Similar observations apply for the void aspect ratio $w_{2}$ and, thus, we omit the corresponding plot. On the other hand, from Fig. 6(b) we observe that the void rotation is substantially different for the two types of loading, since the void orientation angle $\theta$ changes substantially under simple shear, while it remains fixed (aligned with the loading axes) under pure shear. It is also noted for completeness that the 
evolution of the yield stress of the matrix $\sigma_{y}$ for pure shear is very similar with that for simple shear (Fig. 5(d)) and, for this reason, the corresponding comparison for $\sigma_{y}$ is also omitted. More interestingly, we observe from Figs. 6(c) and 6(d) that the stress-strain response of the porous material is almost identical for the two types of loading up to $\bar{E}_{e} \approx 0.3$. At larger strains, the two curves deviate from each other, with the stress-strain curve for pure shear increasing continuously with increasing $\bar{E}_{e}$ and the curve for simple shear exhibiting a softening behavior after a certain strain level. Accordingly, Fig. 6(f) shows that under pure shear the critical condition $\left(H_{J}=0\right)$ for strain localization is never met, while Fig. 6(e) shows that under simple shear the associated critical condition $\left(H_{J}=H_{c r}\right)$ is satisfied at a given strain, at which the material behavior localizes into a shear band. The above observations indicate that the rotation of the voids (on average) has a strong softening effect at large enough strains which ultimately leads to the development of instabilities in the macroscopic behavior of the material.

\subsection{Plane-strain loading at fixed stress triaxiality $X_{\sigma}$}

Figure 7 shows plots of the IVH estimates for the evolution of the internal and macroscopic variables, as a function of the macroscopic equivalent strain $\bar{E}_{e}$, for the non-vanishing spin (continuous curves) and vanishing-spin (dotted curves) loading conditions (22) and (23), respectively. In these plots, the values of $\alpha$ and $\beta$ are allowed to change with the deformation such that the stress triaxiality $X_{\sigma}$ remains fixed throughout the loading process. Results are shown for an initially isotropic distribution of spherical voids with initial porosity $f_{0}=10 \%$, subjected to stress triaxialities $X_{\sigma}=-0.1,0.1,0.3,0.6,1$. Since the evolution of $\sigma_{y}$ for the loading conditions considered here is similar to that discussed earlier for simple shear deformation (Fig. 5(d)), the corresponding plots are omitted for brevity.

High triaxiality $\left(X_{\sigma}=1\right)$. When the porous material is subjected to the relatively large stress triaxiality $X_{\sigma}=1$, under either non-vanishing spin or vanishing-spin loading conditions, we observe that the porosity $f$ (Fig. 7(a)) grows continuously and very rapidly with increasing strain $\bar{E}_{e}$, indicating accordingly a strong softening of the material. The changes of the average shape and orientation of the voids with increasing $\bar{E}_{e}$, as defined by the evolution of the microstructural variables $w_{1}$ (Fig. 7(b)), $w_{2}$ (Fig. 7(c)) and $\theta$ (Fig. 7(d)), are not very 


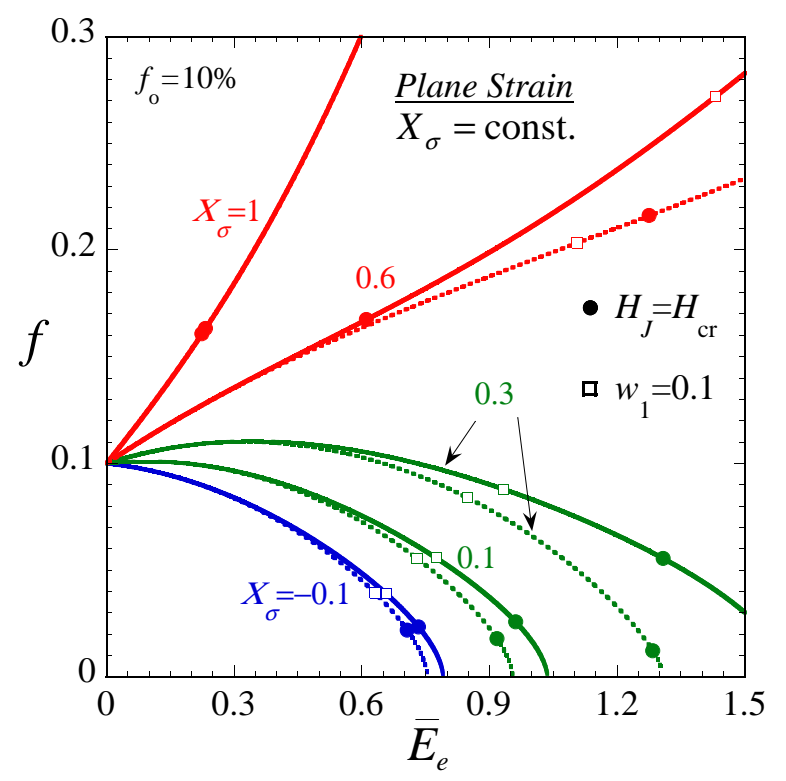

(a)

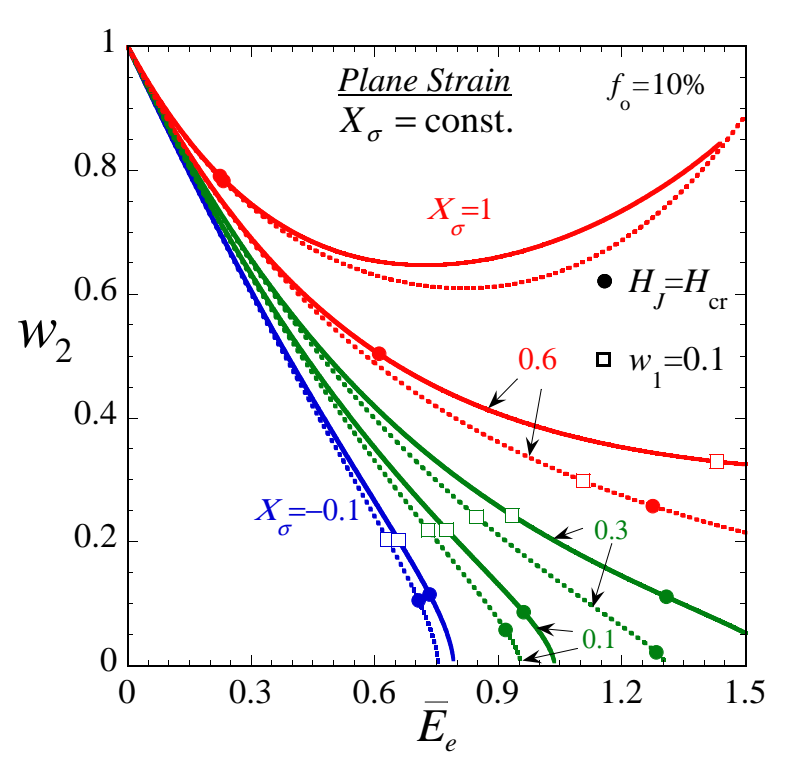

(c)

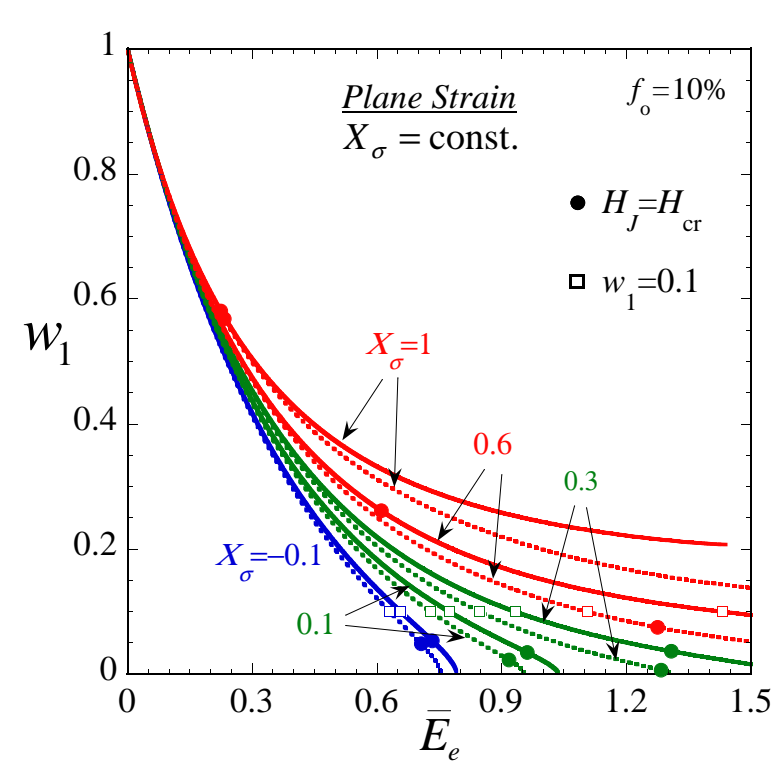

(b)

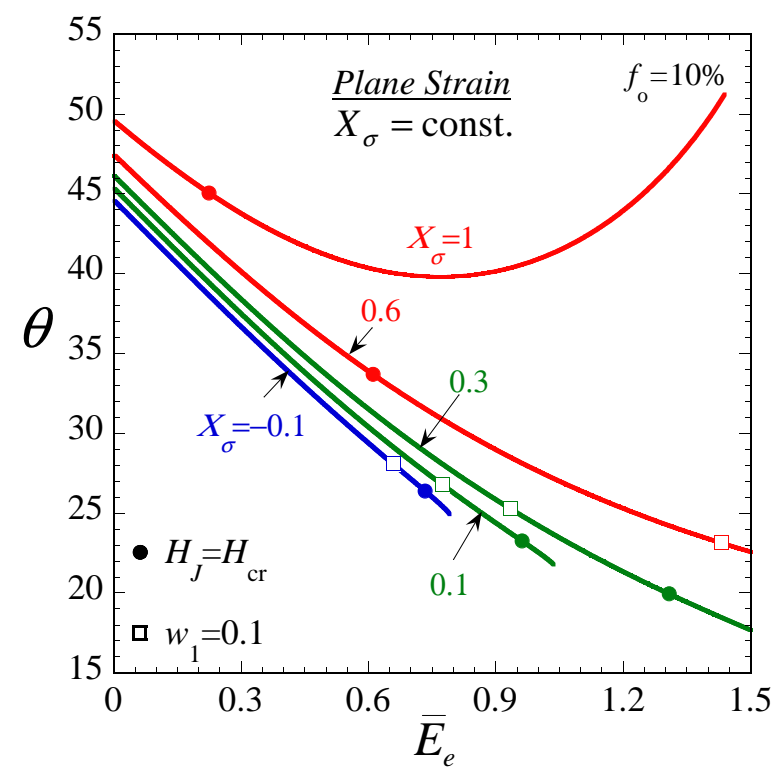

(d)

Figure 7: IVH results for an initially isotropic porous material with $f_{0}=10 \%$ initial porosity subjected to plane strain loading with fixed stress triaxiality $X_{\sigma}$. The evolution of the associated internal and macroscopic variables is shown as a function of the macroscopic equivalent strain $\bar{E}_{e}$ for the cases of non-vanishing spin loadings (continuous curves) and vanishing-spin loadings (dotted curves). (a) Porosity $f$ for both cases. Void aspect ratios (b) $w_{1} \equiv a_{3} / a_{1}$ and (c) $w_{2} \equiv a_{3} / a_{2}$, also for both cases. (d) Void orientation angle $\theta$ for non-vanishing spin loadings. ... 
significant, when compared with the evolution of the porosity $f$. In addition, comparing the results for the evolution of the microstructure under non-vanishing spin (continuous curves) and vanishing-spin (dotted curves) loading, we infer that void rotation has practically no effect on the evolution of the porosity (Fig. 7(a)), although it has some effect at large enough strains on the pore shape change (Figs. 7(b) and 7(c)).

From the results of Figs. 7(e) and 7(g) for the non-vanishing spin loading, we observe that at the early stages of the deformation the macroscopic response of the material exhibits hardening, which is due to the strain hardening of the matrix phase. However, as can be seen from Fig. $7(\mathrm{i})$, the macroscopic hardening modulus $H_{J}$ drops rather quickly, and at some critical value of the strain, it reaches the appropriate critical value $H_{c r}$, indicating the onset of strain localization. Subsequently, the material exhibits continuous softening and the shear and normal components of the stress drop (see Figs. 7(e) and 7(g)). Furthermore, we observe that the evolution of the stress-strain curves (Figs. 7(e) and $7(\mathrm{~g})$ ) and of the hardening modulus (Fig. 7(i)) for the non-vanishing spin loading are almost identical with the corresponding results for the vanishing-spin loading (Figs. $7(\mathrm{f}), 7(\mathrm{~h})$ and $7(\mathrm{j})$ ), which is consistent with our earlier observation that void rotation has a negligible effect on the evolution of the porosity (Fig. 7(a)). The above observations indicate that, under either non-vanishing spin or vanishing-spin conditions, for the loading case $X_{\sigma}=1$ the macroscopic response of the material is dominated by the softening effect due to the growth of the porosity, while the effects of the pore shape and orientation are negligible.

Moderate triaxiality $\left(X_{\sigma}=0.6\right)$. From the results for $X_{\sigma}=0.6$, under either non-vanishing spin or vanishing-spin conditions, we observe that the porosity $f$ (Fig. $7(\mathrm{a})$ ) grows once again, but with smaller growth rate than for the case $X_{\sigma}=1$. Thus, the evolution of the porosity acts again as a softening mechanism, but this time has a weaker effect. The void aspect ratio $w_{1}$ (Fig. $\left.7(\mathrm{~b})\right)$ decreases gradually with increasing strain $\bar{E}_{e}$, as for the case $X_{\sigma}=1$, but at a faster rate. On the other hand, the evolution of both $w_{2}$ (Fig. $7(\mathrm{c})$ ) and $\theta$ (Fig. $7(\mathrm{~d})$ ) is significantly different for $X_{\sigma}=0.6$ than for $X_{\sigma}=1$, with $w_{2}$ and $\theta$ evolving faster and reaching smaller values in the present case. Comparing the evolution of the microstructural variables $f, w_{1}$ and $w_{2}$ for non-vanishing spin and vanishing-spin loadings 
(with $X_{\sigma}=0.6$ ), it may be inferred that void rotation has the effect of accelerating void growth (Fig. 7(a)) and decelerating the void shape change (Figs. 7(b) and 7(c)).

From the results of Figs. 7(e) and 7(g) for non-vanishing spins, we observe that the initial hardening regime of the macroscopic response for the case $X_{\sigma}=0.6$ extends to larger strains than for $X_{\sigma}=1$, while from Fig. 7(i) we observe that the drop of the hardening modulus $H_{J}$ is slower for $X_{\sigma}=0.6$ and, accordingly, the onset of strain localization occurs at a larger strain. Note that both these observations are consistent with our earlier observation that the softening effect due to the porosity growth is weaker for $X_{\sigma}=0.6$ than for $X_{\sigma}=1$ (see Figs. 7(a)). Quite interestingly, we observe from Fig. 7(j) that the critical strain at the onset of localization for the case of the vanishing-spin loading is more than twice that for the case of the non-vanishing spin loading (see Fig. 7(i)). Similar to the case of simple shear loading discussed in subsection 4.1, this observation for $X_{\sigma}=0.6$ suggests that void rotation may have a dramatic destabilizing effect on the macroscopic response of the material. In addition, it should be remarked that, for the non-vanishing spin conditions, localization takes place while the void shape is still quite rounded (the average aspect ratio $w_{1}$ reaching a value of 0.1 at nearly twice the critical strain). On the other hand, for the vanishing-spin conditions, the localization condition is met much later when the void shape is already quite flat and contact of the void faces may have already taken place. This suggests that shear localization may indeed be more physically relevant for non-vanishing spin conditions, where void rotations are significant, than for vanishing-spin conditions, where void rotations are not expected.

Small triaxiality $\left(X_{\sigma}=0.3\right)$. The evolution of the porosity $f\left(\right.$ Fig. $7($ a $)$ ) for $X_{\sigma}=0.3$ is substantially different from that for the cases $X_{\sigma}=0.6$ and $X_{\sigma}=1$, discussed above. In particular, for $X_{\sigma}=0.3$, we observe that the porosity $f$ increases slightly up to a certain strain and then decreases continuously and tends to zero with increasing $\bar{E}_{e}$, indicating that the porosity evolution eventually plays the role of a hardening mechanism in this case. Furthermore, we observe that the aspect ratios $w_{1}$ and $w_{2}$ (Figs. $7(\mathrm{~b})$ and $7(\mathrm{c})$ ) decrease faster for $X_{\sigma}=0.3$ than for $X_{\sigma}=0.6$ and go to zero at the same strain that $f$ goes to zero, indicating a propensity of the voids to collapse to micro-cracks. In addition, we observe that the rotation of the voids, as defined by the evolution of the angle $\theta$ (Fig. $7(\mathrm{~d})$ ), is only 


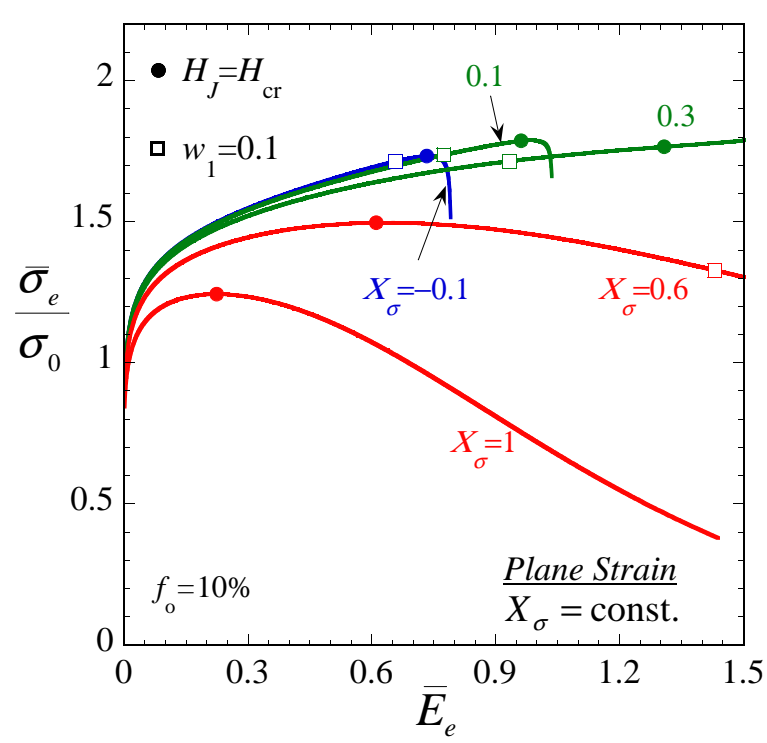

(e)

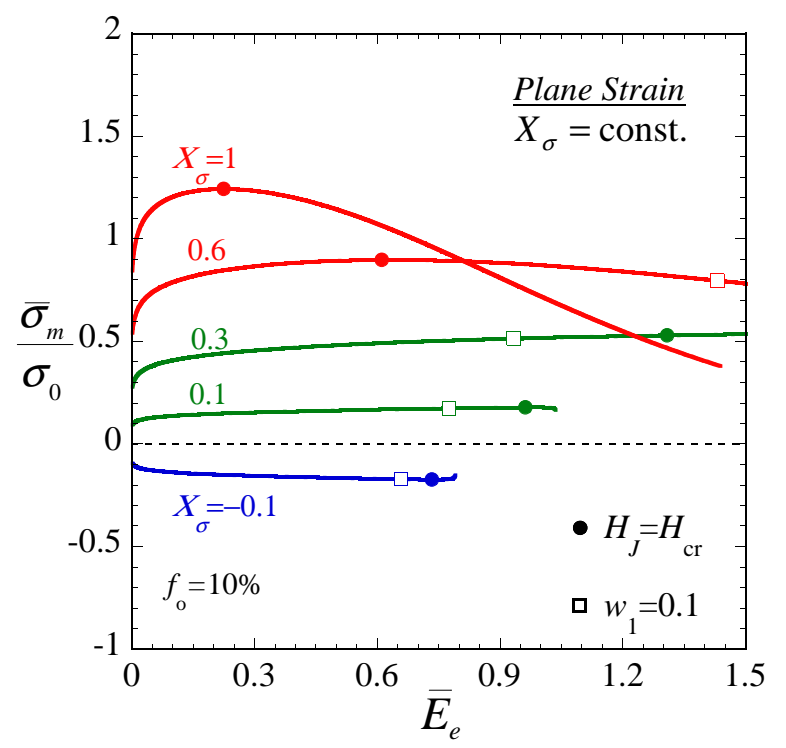

$(\mathrm{g})$

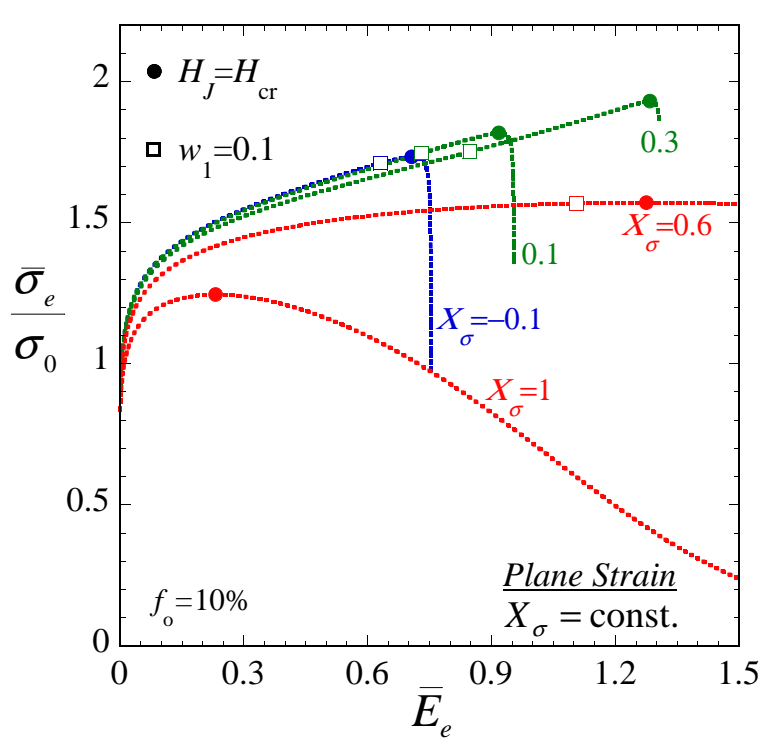

(f)

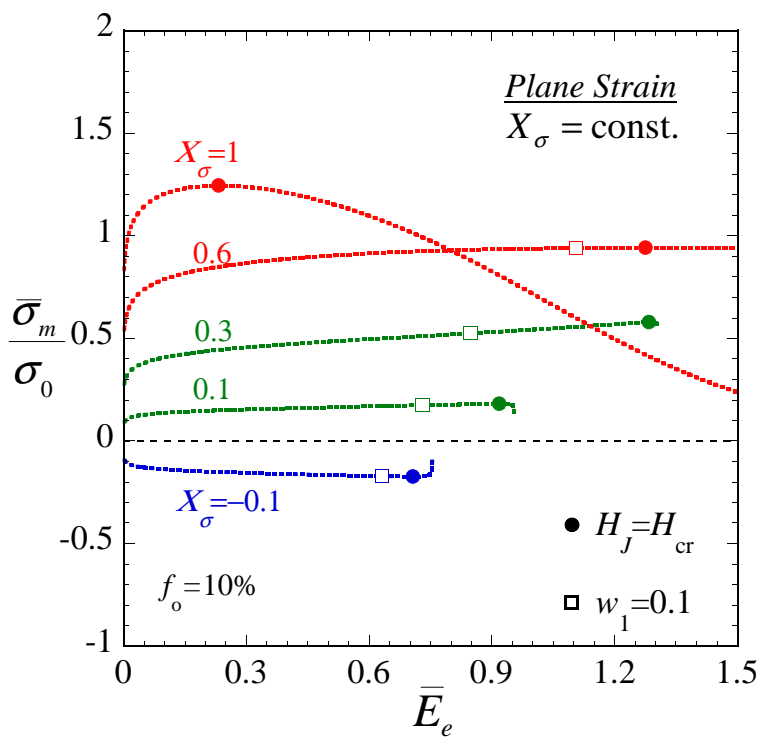

(h)

Figure 7: ... Average equivalent stress $\bar{\sigma}_{e}$ for (e) non-vanishing spin loadings and (f) vanishing-spin loadings. Average mean stress $\bar{\sigma}_{m}$ for $(\mathrm{g})$ non-vanishing spin loadings and (h) vanishing-spin loadings. ... 


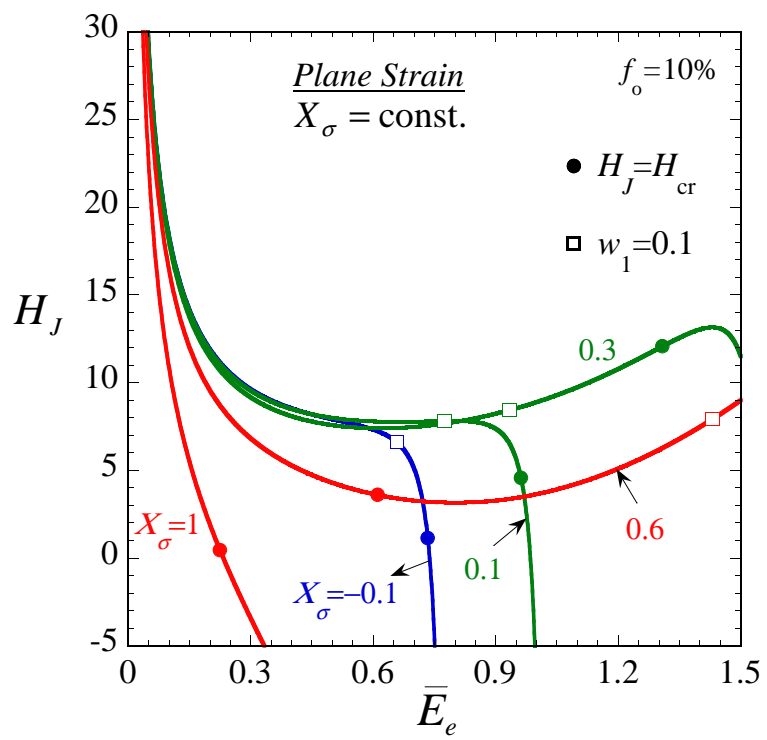

(i)

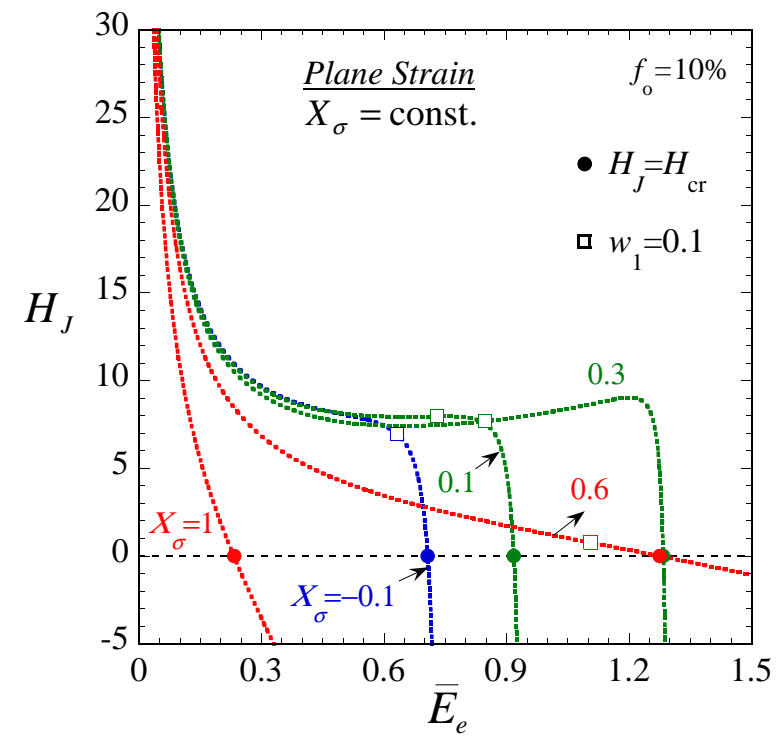

(j)

Figure 7: ... Macroscopic Jaumann hardening modulus $H_{J}$ for (i) non-vanishing spin and (j) vanishing-spin loadings.

slightly faster for $X_{\sigma}=0.3$ than for $X_{\sigma}=0.6$. Comparing the evolution of the porosity $f$ (Fig. $7(\mathrm{a}))$ and aspect ratios $w_{1}$ (Fig. 7(b)) and $w_{2}$ (Fig. 7(c)) under non-vanishing spin and vanishing-spin loading, we observe that void rotation has the effect of decelerating the void collapse rate, i.e., the variables $f, w_{1}$ and $w_{2}$ go to zero at a larger strain for the non-vanishing spin loading.

The macroscopic stress-strain curves in Fig. 7(e) show that the material response is harder for $X_{\sigma}=0.3$ than for $X_{\sigma}=0.6$, which is consistent with the fact that the evolution of the porosity (Fig. 7(a)) acts as a hardening mechanism (at large enough strains) in the former case and as a softening mechanism in the latter. In addition, comparing the stressstrain curves for non-vanishing spin loading (Figs. 7(e) and 7(g)) with the corresponding curves for vanishing-spin loading (Figs. $7(\mathrm{f})$ and $7(\mathrm{~h})$ ), we observe that the macroscopic behavior of the porous metal is slightly softer for the former than for the later type of loading, which is consistent with the fact that the slower reduction of the porosity in the former case (see Fig. 7(a)) leads to a weaker hardening behavior of the material.

The most remarkable feature of the results shown in Fig. 7 for $X_{\sigma}=0.3$, for both the 
non-vanishing spin and the vanishing-spin loading, is that the macroscopic behavior of the material becomes unstable at sufficiently large strains, despite the hardening induced by the reduction of the porosity (Fig. 7(a)). As argued by Agoras and Ponte Castañeda (2014) in the context of vanishing-spin loading conditions with fixed strain triaxiality, the basic mechanism responsible for this instability is the collapse of the voids, which, at the critical strain, produces sufficiently strong softening to overcome the corresponding hardening effects due to the reduction of the porosity and the strain hardening of the matrix phase. Note that for the non-vanishing spin loading the instability occurs at a slightly larger strain than for the vanishing-spin loading, suggesting that for the loading case $X_{\sigma}=0.3$ void rotation may have a (weak) hardening effect, which is consistent with the fact that the void collapse rate for the non-vanishing spin loading is slower (i.e., the aspect ratios $w_{1}$ and $w_{2}$ in Figs. $7(\mathrm{~b})$ and $7(\mathrm{c})$ decrease with slower rates) than for the vanishing-spin loading.

In contrast with the loading cases $X_{\sigma}=0.6$ and $X_{\sigma}=1$, we observe that for $X_{\sigma}=0.3$ the onset of strain localization takes place for relatively small aspect ratios (e.g., under the non-vanishing spin loading, $w_{1} \approx 0.05$ and $w_{2} \approx 0.1$ ). As already mentioned, the relevance of this prediction could be questioned on the basis of the fact that void surface contact is expected to take place earlier and to have a hardening effect that could ultimately prevent strain localization (Hutchinson and Tvergaard, 2012). In this connection, however, it should be remarked that the recent (unit cell) numerical analysis by Tvergaard (2014) on the development of shear localization instabilities in elastic-plastic porous materials has shown that void surface contact delays the onset of localization, but it does not completely eliminate it. Although quantitative comparisons of the predictions of the IVH model with results from the latter work can not be made due to differences in the microstructural features and loading conditions considered, it is interesting to remark that, at least for the case of the non-vanishing spin loading, the predictions of the IVH model for relatively small stress triaxialities (e.g., $X_{\sigma}=0.3$ ) appear to be qualitatively consistent with the corresponding numerical results of Tvergaard (2014) for shear localization, as well as for the propensity of the voids to collapse to micro-cracks, which in the context of the IVH model provides the required softening mechanism for strain localization. 
Very small and negative triaxialities $\left(X_{\sigma}=0.1,-0.1\right)$. The evolution of the internal and macroscopic variables in Fig. 7 for the stress triaxialities $X_{\sigma}=0.1$ and -0.1 is qualitatively similar with that for the loading case $X_{\sigma}=0.3$, discussed above. Note, however, that for smaller values of $X_{\sigma}$ the voids collapse to micro-cracks faster, i.e., the variables $f$ (Fig. $7(\mathrm{a})), w_{1}$ (Fig. $\left.7(\mathrm{~b})\right)$ and $w_{2}$ (Fig. $\left.7(\mathrm{c})\right)$ drop to zero faster with decreasing values of $X_{\sigma}$ (for $\left.X_{\sigma} \leq 0.3\right)$. As a result, void closure $(f=0)$ occurs at smaller strains, while the softening effect due to void collapse becomes stronger for smaller values of $X_{\sigma}$, thus leading to instabilities at smaller strains. The differences between the results for the non-vanishing spin (continuous curves) and vanishing-spin (dotted curves) loading, for any given variable in Fig. 7, decrease with decreasing $X_{\sigma}$. Results for stress triaxialies smaller than -0.1 (not included in Fig. 7) show that there are practically no differences between the two types of loading, suggesting that void rotations play no role on the macroscopic response of porous materials at such levels of triaxiality.

\subsection{Failure curves}

We conclude this section with a discussion of the effect of the stress triaxiality on the ductile failure of porous materials, as defined by the onset of macroscopic strain localization. To this end, Fig. 8 shows plots of the critical equivalent strain $\bar{E}_{e}^{c r}$ at the onset of localization as a function of the applied stress triaxiality $X_{\sigma}$. Fig. 8(a) presents results for the nonvanishing spin loading (22) with fixed $X_{\sigma}$, for $f_{0}=1 \%, 10 \%$ and $30 \%$ initial porosity, while Fig. 8(b) compares the failure curve for $f_{0}=10 \%$ of Fig. 8(a) with corresponding results for the vanishing-spin loading (23) with fixed $X_{\sigma}$. The dashed portion of each failure curve in Fig. 8 corresponds to instabilities occurring for void aspect ratios $w_{1}^{c r}<0.1\left(w_{2}^{c r}>w_{1}^{c r}\right)$. In this connection, we recall that under loading conditions leading to the collapse $\left(w_{1}, w_{2} \rightarrow 0\right)$ of the voids, partial contact of the opposite faces of the voids is expected to take place prior to complete void closure (Hutchinson and Tvergaard, 2012) and, thus, the approximation that the void shape remains ellipsoidal is expected to become invalid for sufficiently small values of the void aspect ratios. It should be emphasized, however, that the ellipsoidal poreshape assumption adopted by the IVH model refers not to the shape of any individual void but to the shape of the voids on average. Thus, it could be argued that partial contact 
of opposite faces in any given void is equivalent to splitting the void into two (or more) voids, the average shape of which may still be (approximately) described by a characteristic ellipsoid. For this reason, it is plausible that the average ellipsoidal-shape assumption may still be physically relevant even after contact of the void faces has occurred in individual voids. In other words, despite the fact that the IVH model does not account for void surface contact, the instabilities of Fig. 8 for $w_{1}^{c r}<0.1$ may still be physically relevant, albeit at perhaps higher critical strains.

From the results of Fig. 8(a), we observe that the failure curves exhibit a maximum at a certain value $X_{\sigma}^{*}$ of the stress triaxiality $X_{\sigma}$, which for porosities $f_{0}=10 \%$ and $30 \%$ is $X_{\sigma}^{*} \approx 0.3$ and for $f_{0}=1 \%$ is $X_{\sigma}^{*} \approx 0.4$. For any given $f_{0}$, we observe that the material becomes progressively more unstable (i.e., the critical strain $\bar{E}_{e}^{c r}$ becomes smaller) with either increasing or decreasing $X_{\sigma}$ from $X_{\sigma}=X_{\sigma}^{*}$. This behavior may be understood to be a consequence of the fact that the effect of the underlying softening mechanisms that are responsible for failure become progressivey stronger with either increasing or decreasing $X_{\sigma}$ from $X_{\sigma}=X_{\sigma}^{*}$. In this connection, we recall from the results of the previous subsection for $f_{0}=10 \%$ that the dominant failure mechanism in the range of stress triaxialities $X_{\sigma}<0.3$ is void collapse, while in the range $X_{\sigma}>1$ it is void growth.

The failure curves of Fig. 8(b) for non-vanishing spin and vanishing-spin loadings are very similar for stress triaxialities in the regions $X_{\sigma}<0.3$ and $X_{\sigma}>1$, accordingly suggesting that the effect of void rotation is relatively weak for loadings with $X_{\sigma}<0.3$ or $X_{\sigma}>1$. On the other hand, for values $0.3 \lesssim X_{\sigma} \lesssim 1$ we observe that the failure curves of Fig. 8(b) are substantially different, indicating that the effect of void rotation is rather significant in this intermediate range. In particular, note that for the vanishing-spin loading the critical strain $\bar{E}_{e}^{c r} \rightarrow \infty$ as $X_{\sigma} \rightarrow 0.5$, while for the non-vanishing spin loading $\bar{E}_{e}^{c r} \approx 0.8$ for $X_{\sigma}=0.5$. This is similar to the pure shear versus simple shear comparison, where the critical strain $\bar{E}_{e}^{c r} \rightarrow \infty$ in the first case, and $\bar{E}_{e}^{c r} \approx 1$ in the second.

Finally, it should be recalled in the context of Fig. 8 that, for given initial porosity $f_{0}$, there is a range of stress triaxialities within which strain localization is predicted to occur for substantially distorted pore shapes, i.e., for pore shapes characterized by an aspect ratio $w_{1}^{c r}<0.1$ (see dashed portions of the curves in Fig. 8). As discussed earlier, the theoretical 


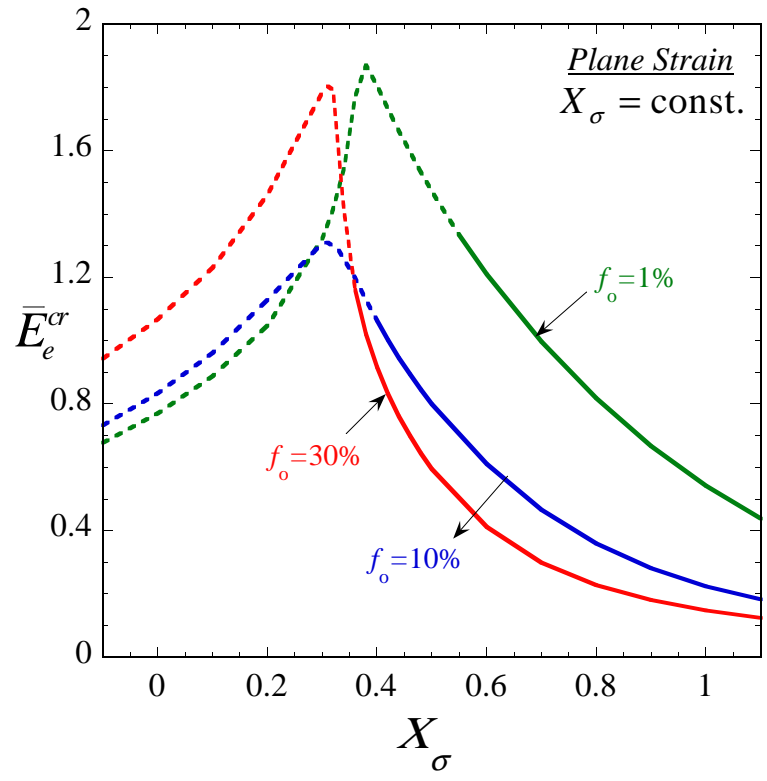

(a)

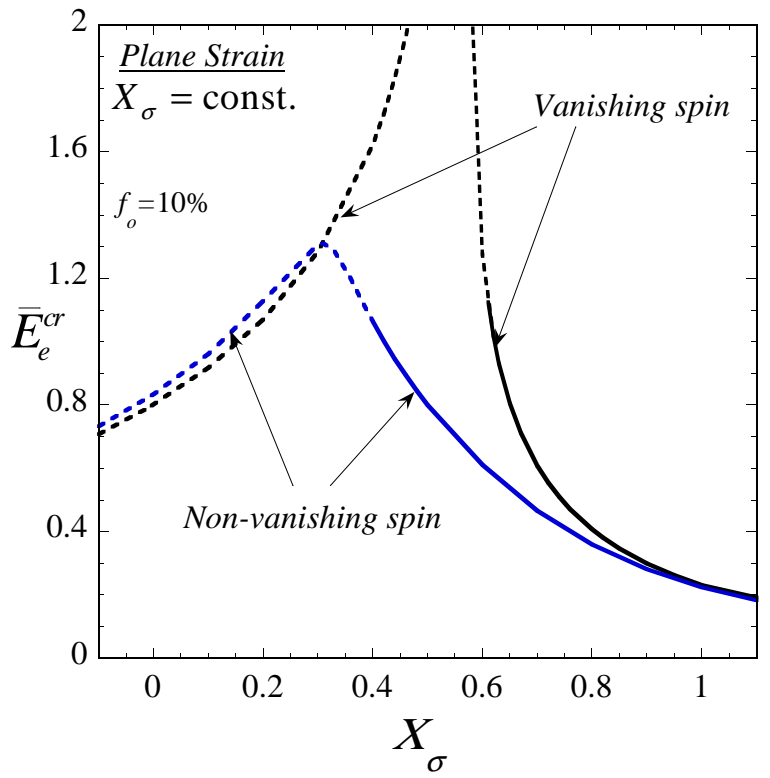

(b)

Figure 8: (a) The critical strain $\bar{E}_{e}^{c r}$ at the onset of macroscopic localization in initially isotropic porous materials subjected to non-vanishing spin loading of the type (22) with fixed stress triaxiality $X_{\sigma}$, is plotted as a function of $X_{\sigma}$, for $f_{0}=1 \%, 10 \%$ and $30 \%$. (b) The failure curve of Part (a) for $f_{0}=10 \%$ is compared with corresponding results for vanishing-spin loading of the type (23) with fixed $X_{\sigma}$. The dashed portion of each failure curve corresponds to instabilities occurring at a void aspect ratio $w_{1}<0.1$. 
predictions for the instabilities in this range of stress triaxialities could be affected by contact of the void surfaces (especially for small initial porosities), or by the presence of hard particles (when the voids nucleate from such hard particles). However, at least for non-vanishing spin loadings and for the case of pre-existing voids, the recent work by Tvergaard (2014) suggests that contact of the void surfaces may not be enough to prevent shear localization, although contact of the void surfaces could delay the onset of the localization, relative to the predictions of the IVH model (which ignores contact of the void surfaces). On the other hand, when there is no pre-existing porosity and the voids nucleate from hard particles (as is the case, for example, in the experimental work of Barsoum and Faleskog, 2007), it would be expected that such particles would significantly affect the localization predictions by effectively shutting down the void collapse mechanism that is responsible for the geometric softening at the low stress triaxialities. Thus, the physical relevance of the theoretical predictions of the model for shear localization at low triaxialities would be expected to depend on the specific source of the porosity (pre-existing versus nucleating at hard particles). In addition, it should be emphasized that the predictions of the model discussed in this work are for plane strain conditions, and therefore the cannot be directly compared with the combined tension-torsion experimental results of Barsoum and Faleskog (2007) and Haltom et al. (2013), which are for plane stress conditions.

\section{Concluding remarks}

In this paper, we have extended the constitutive model of Agoras and Ponte Castañeda (2013, 2014) for porous rigid-plastic materials to account for void rotations under general finite-deformation loading conditions. The model makes use of the iterated variational linear comparison homogenization (IVH) method developed by Ponte Castañeda (2012) to characterize the instantaneous macroscopic response of porous metals by means of yield surfaces depending on the current values of the porosity, the average shape and orientation of the pores, as well as the shape and orientation of the distribution function for the centers of the pores. In addition, consistently derived homogenization estimates for the average strain-rate and vorticity in the pores are used to derive corresponding evolution equations for the porosity, average shape and orientation of the voids (and their distribution). In its final form, 
the IVH model can be regarded as a standard "internal variable" plasticity model, where the evolution of the microstructural variables accounts for "geometrical" contributions to the overall hardening/softening of the porous material, adding to the usual "constitutive" contributions already accounted for through the evolution of the yield stress in the solid matrix material.

The model was first applied to investigate the macroscopic response of initially isotropic rigid-plastic porous metals under simple shear loading. It was found that significant stress triaxialities develop in the material as a result of a strong in-plane anisotropy that is induced by the change in the pore average shape from spherical to flat ellipsoids. This finding is in qualitatively agreement with earlier results of Kailasam and Ponte Castañeda (1997) and Danas and Ponte Castañeda (2009a), but quite different from the prediction of Gurson-type models (e.g., Nahshon and Hutchinson, 2008), where the material is assumed to remain isotropic, so that no normal stresses (or stress triaxiality) can develop under simple shear loading. In addition, strain localization was predicted to take place in a shear band parallel to the shear plane of the loading. By means of suitable comparisons with the corresponding results for pure shear loading - where the voids undergo no rotation on average and no shear localization occurs - we deduced that the rotation of the voids for simple shear loading conditions has a strong softening effect, which tends to dramatically enhance the susceptibility of the material to fail by shear localization. In this context, it is crucial to note that the average rotation of the voids is a consequence of non-vanishing macroscopic spin associated with simple shear loading conditions, since the vanishing-spin conditions associated with pure shear loading for initially isotropic distributions of spherical voids would produce no void rotations (on average).

Next, the macroscopic response of the porous metal was investigated for combined plane strain tension and shear with prescribed, fixed stress triaxiality $X_{\sigma}$. It was found that the stress triaxiality has a strong effect on the evolution of the porosity and average shape of the pores, which in turn has a very significant effect on both the shear and normal components of the macroscopic stress, leading to the development of shear localization at sufficiently large strains. In particular, consistent with earlier observations (Agoras and Ponte Castañeda, 2014), the main failure mechanism is found to be void growth at large stress 
triaxialities $\left(X_{\sigma} \gtrsim 1\right)$ and void collapse at small stress triaxialities $\left(X_{\sigma} \lesssim 0.3\right)$, such that the localization strain decreases both with increasing triaxialities at the larger triaxialities, and with decreasing triaxialities at the lower triaxialities. For these ranges of triaxialities, voids rotations were found to have no significant effects on the macroscopic hardening of the material and therefore no implications for shear localization. On the other hand, for intermediate values of $X_{\sigma}\left(0.3 \lesssim X_{\sigma} \lesssim 1\right)$, it was found that void rotations tend to destabilize the macroscopic response of the porous materials leading to much lower localization strains than when void rotations are not present (as in biaxial straining of the material).

This stronger susceptibility to failure by shear localization for shearing loading conditions (i.e., with non-vanishing spin) would appear to be consistent with recent finite element simulation results (Tvergaard, 2014) for porous plastic media with periodic microstructures (although great care should be exercised when comparing instability results for random and periodic microstructures, due to the great sensitivity of these results to the initial distribution of the pores as determined, for example, by the shape of the unit cell in the periodic context). In this context, it is also important to recall that the IVH model does not take into account the possible contact of void surfaces (Hutchinson and Tvergaard, 2012) at sufficiently low stress triaxialities. However, while contact of the void faces is expected to have a hardening effect on the macroscopic response, which may indeed slow down the possible development of shear localization instabilities, the finite element results of Tvergaard (2014) show that at least for shearing loading conditions contact of the void faces is not enough to completely stop the development of shear bands. In this sense, our results are at the very least qualitatively consistent with the FEM results in that porous materials are predicted to be more susceptible to failure by shear localization for "shearing" loading conditions leading to non-vanishing spins of the voids than for "axial" loading conditions where no void rotations are expected. In addition, it should be noted that when the porosity nucleates from the de-bonding of hard particles, such particles can have significant implications for the evolution of the porosity at low triaxialities, since the presence of the particles would be expected to slow down, or completely shut down, the void collapse mechanism responsible for the shear localization predictions at low triaxialities. For these reasons, the predictions of the model for shear localization at the lower triaxialities need to be carefully evaluated in the context of the 
specific details of the material and loading conditions, as they may require modification when other mechanisms not yet accounted for by the model, such as contact of the void faces, or pinning of the voids by hard particles, are found to play a role.

In conclusion, the results of this paper suggest an additional level of complexity in modeling the macroscopic response of porous plastic materials. While experimental results have already shown that the stress triaxiality does not suffice to characterize the failure of porous metals at low stress triaxialities, and other measures such as the Lode parameter must be introduced, the results of this paper strongly suggest that even the triaxiality and the Lode parameter may not be enough to completely describe failure of porous metals in the low to moderate stress triaxiality regime. Indeed, the results of this work show that the macroscopic spin, or vorticity can have strong implications for shear localization at intermediate values of the triaxiality. In particular, this means that caution must be exercised when comparing experimental results based on different experimental conditions (e.g., different test specimens). In addition, this also suggests that to really be able to model and predict failure of porous plastic materials it may be necessary to implement a more microscopic point of view, since these failure mechanisms appear to ultimately be controlled by microscopic features such as the evolution of the average size, shape and orientation of the voids. While macroscopic stress measures such as the triaxiality and the Lode parameter certainly have implications for failure on porous ductile materials, their effects are only indirect - through the corresponding effects on the evolution of the microstructure. In this sense, it would seem necessary to directly account for such microstructural evolution effects, something that could only be accomplished with more sophisticated constitutive models accounting for additional microstructural variables beyond the porosity. It is precisely for these reasons that homogenization models, such as the one developed in the context of this work, have a distinct advantage relative to the more empirical Gurson-type models, since they allow the direct incorporation of appropriate statistical variables, such as the average void shape and orientation, to more accurately account for the state and anisotropic evolution of the porous microstructure. In addition, these homogenization models can be generalized in a seamless fashion to account for additional microstructural features, such as crystallographic texture, whose evolution is known to be crucial in modeling the response of certain low-symmetry 
materials, such as magnesium and polar ice. Thus, the techniques developed in this work could be generalized to model the combined effect of porosity and crystallographic texture in porous low-symmetry polycrystals. A first step in that direction is provided by the recent work by Lebensohn et al. (2011), where a less sophisticated homogenization method was used to determine macroscopic yield surfaces of porous polycrystals.

\section{Acknowledgements}

This work has been supported in part by the National Science Foundation under grant CMMI-1332965.

\section{Appendix: Detailed expressions for the model}

In this Appendix, we provide the required detailed expressions for the IVH model, referring to Agoras and Ponte Castañeda (2013) and Agoras and Ponte Castañeda (2014) for their derivations. In this context, it is important to recall that these expressions are obtained by means of the (PCW) estimates of Ponte Castañeda and Willis (1995) for the LCC at each iteration step. In addition, use is made of the results of Idiart and Ponte Castañeda (2007) to relate the averages and second moments of the fields in the matrix and vacuous phases of the LCC to the corresponding quantities in the actual nonlinear porous material.

We begin by recalling that the normalized effective viscous compliance tensors $\widehat{\mathbb{M}}[i]$ the $i$-level LCC is given by

$$
\begin{aligned}
\widehat{\mathbb{M}}_{[1]}^{P C W} & =\mathbb{K}+c_{[1]}^{(2)}\left[\hat{\mathbb{Q}}^{(p)}-c_{[1]}^{(2)} \hat{\mathbb{Q}}^{(d)}\right]^{-1}, \\
\widehat{\mathbb{M}}_{[i]}^{P C W} & =r_{[i]}^{-1} \mathbb{K}+r_{[i]}^{-1} c_{[i]}^{(2)}\left[\left(r_{[i]} \widehat{\mathbb{M}}_{[i-1]}^{P C W}-\mathbb{K}\right)^{-1}+\left(1-c_{[i]}^{(2)}\right) \hat{\mathbb{Q}}^{(d)}\right]^{-1} \\
& \equiv \widehat{\mathbb{M}}_{[i]}^{P C W}\left(r_{[2]}, \ldots, r_{[i]}\right), \quad i=2, \ldots, N
\end{aligned}
$$

where $\mathbb{K}$ is the fourth-order identity tensor in the deviatoric space, the variables $c_{[i]}^{(2)}(i=$ $1, \ldots, N)$ are incremental volume fractions, the variables $r_{[i]}(i=2, \ldots, N)$ are normalized shear moduli in the LCC (see Agoras and Ponte Castañeda, 2014 for details), $\hat{\mathbb{Q}}^{(p)}$ and $\hat{\mathbb{Q}}^{(d)}$ are the fourth-order microstructural tensors, accounting for the shape and orientation of the pores and their distribution, respectively. In this Appendix, it is assumed for generality that 
the shape and orientation of the pores and distribution may be different. However, in the body of the paper, and in all the results presented therein, it has been assumed that the shape and orientation of the pores are identical to those of their distribution, and hence that $\hat{\mathbb{Q}}^{(p)}=\hat{\mathbb{Q}}^{(d)}=\hat{\mathbb{Q}}\left(w_{\alpha}, \mathbf{n}_{i}\right)$. In general, these microstructural tensors have to be computed numerically, as shown in the work of Aravas and Ponte Castañeda (2004).

For the rigid plastic porous metal, the second moment of the equivalent stress field over the matrix phase $\left(\overline{\bar{\sigma}}_{e}^{(1)}\right)^{2}$ can be shown to satisfy the relations (Agoras and Ponte Castañeda, 2014)

$$
\begin{aligned}
\left(\overline{\bar{\sigma}}_{e}^{(1)}\right)^{2} & =\frac{3}{2} \frac{1}{1-c_{[1]}^{(2)}} \overline{\boldsymbol{\sigma}} \cdot\left[\prod_{j=2}^{N} \mathbb{B}_{[j]}^{(2)}\right]^{T} \widehat{\mathbb{M}}_{[1]}^{P C W}\left[\prod_{j=2}^{N} \mathbb{B}_{[j]}^{(2)}\right] \overline{\boldsymbol{\sigma}} \equiv \mathcal{F}_{[1]}\left(r_{[2]}, \ldots, r_{[N]}\right), \\
\left(\overline{\bar{\sigma}}_{e}^{(1)}\right)^{2} & =\frac{3}{2} \frac{r_{[i]}}{1-c_{[i]}^{(2)}} \overline{\boldsymbol{\sigma}} \cdot\left[\prod_{j=i+1}^{N} \mathbb{B}_{[j]}^{(2)}\right]^{T}\left[\widehat{\mathbb{M}}_{[i]}^{P C W}-c_{[i]}^{(2)}\left(\mathbb{B}_{[i]}^{(2)}\right)^{T} \widehat{\mathbb{M}}_{[i-1]}^{P C W} \mathbb{B}_{[i]}^{(2)}\right]\left[\prod_{j=i+1}^{N} \mathbb{B}_{[j]}^{(2)}\right] \overline{\boldsymbol{\sigma}} \\
& \equiv \mathcal{F}_{[i]}\left(r_{[2]}, \ldots, r_{[N]}\right), \quad i=2, \ldots, N
\end{aligned}
$$

where the corresponding stress concentration tensors are provided by

$$
\mathbb{B}_{[i]}^{(2)}=\left[\mathbb{I}+\left(1-c_{[i]}^{(2)}\right) \hat{\mathbb{Q}}^{(d)}\left(r_{[i]} \widehat{\mathbb{M}}_{[i-1]}^{P C W}-\mathbb{K}\right)\right]^{-1} \equiv \mathbb{B}_{[i]}^{(2)}\left(r_{[2]}, \ldots, r_{[i]}\right), \quad i=2, \ldots, N
$$

In this last expression, $\mathbb{I}$ is the fully symmetric, fourth-order identity tensor. As mentioned in section 2.2, for given applied loading $\overline{\boldsymbol{\sigma}}$ and internal variables $\mathbf{s}$ of the porous metal, the $N$ unknowns $\overline{\bar{\sigma}}_{e}^{(1)}$ and $r_{[i]}(i=2, \ldots, N)$ can be fully determined from the system of $N$ nonlinear equations (29).

The iterated estimates of the PCW type for the average strain-rate in the voids is given by $\overline{\mathbf{D}}^{(2)}=\mathbb{A}^{(2)} \overline{\mathbf{D}}$, where $\mathbb{A}^{(2)}$ is the associated strain-rate concentration tensor, determined by (Agoras and Ponte Castañeda, 2013; Agoras and Ponte Castañeda, 2014)

$$
\mathbb{A}^{(2)}=\prod_{i=1}^{N} \mathbb{A}_{[i]}^{(2)} \equiv \mathbb{A}^{(2)}\left(r_{[2]}, \ldots, r_{[N]}\right),
$$

with

$$
\begin{aligned}
\mathbb{A}_{[1]}^{(2)} & =\left[c_{[1]}^{(2)} \mathbb{I}+\mathbb{K}\left(\hat{\mathbb{Q}}^{(p)}-c_{[1]}^{(2)} \hat{\mathbb{Q}}^{(d)}\right)\right]^{-1}, \\
\mathbb{A}_{[i]}^{(2)} & =\left[c_{[i]}^{(2)} \mathbb{I}+\left(1-c_{[i]}^{(2)}\right)\left[\left(\mathbb{K}-\mathbb{K} \hat{\mathbb{Q}}^{(d)} \mathbb{K}\right)\left(r_{[i]} \widehat{\mathbb{M}}_{[i-1]}^{P C W}\right)^{-1}+\mathbb{K}_{\hat{Q}^{(d)}}\right]\right]^{-1} \\
& \equiv \mathbb{A}_{[i]}^{(2)}\left(r_{[2]}, \ldots, r_{[i]}\right), \quad i=2, \ldots, N .
\end{aligned}
$$


It should be noted that $\mathbb{A}_{[1]}^{(2)}$, which corresponds to the strain-rate concentration tensor at the first level LCC, depends on the shape and orientation of both the pores and distribution, through the microstructural tensors $\hat{\mathbb{Q}}^{(p)}$ and $\hat{\mathbb{Q}}^{(d)}$; however, the other strain-rate concentration tensors $\mathbb{A}_{[i]}^{(2)}(i=2, \ldots, N)$, which correspond to higher level LCCs, only depend on the shape and orientation of the pore distribution, through the tensor $\hat{\mathbb{Q}}^{(d)}$.

Similarly, the average spin tensor of the voids can be obtained iteratively from the corresponding estimates of the PCW type for the LCC at each increment, and is given by

$$
\overline{\mathbf{W}}^{(2)}=\overline{\mathbf{W}}-\mathbb{C}^{(2)} \overline{\mathbf{D}}
$$

where $\mathbb{C}^{(2)}$ is the associated spin concentration tensor in the voids, determined by

$$
\mathbb{C}^{(2)}=\sum_{i=1}^{N}\left[\mathbb{C}_{[i]}^{(2)}\left(\prod_{j=i+1}^{N} \mathbb{A}_{[j]}^{(2)}\right)\right]
$$

with

$$
\begin{aligned}
\mathbb{C}_{[1]}^{(2)} & =-\left(\boldsymbol{\Pi}^{(p)}-c_{[1]}^{(2)} \boldsymbol{\Pi}^{(d)}\right) \mathbb{A}_{[1]}^{(2)}, \\
\mathbb{C}_{[i]}^{(2)} & =\left(1-c_{[i]}^{(2)}\right) \boldsymbol{\Pi}^{(d)}\left[\mathbb{K}\left(r_{[i]} \widehat{\mathbb{M}}_{[i-1]}^{P C W}\right)^{-1}-\mathbb{I}\right] \mathbb{A}_{[i]}^{(2)} \\
& \equiv \mathbb{C}_{[i]}^{(2)}\left(r_{[2]}, \ldots, r_{[i]}\right), \quad i=2, \ldots, N .
\end{aligned}
$$

Note that $\boldsymbol{\Pi}^{(p)}$ and $\boldsymbol{\Pi}^{(d)}$ are the fourth-order Eshelby microstructural tensors, which correspond to the rotation of pores and their distribution, respectively. In the present work, where the shape and orientation of the pores are identical to that of their distribution, $\boldsymbol{\Pi}^{(p)}=\boldsymbol{\Pi}^{(d)}=\boldsymbol{\Pi}\left(w_{\alpha}, \mathbf{n}_{i}\right)$. The tensor $\boldsymbol{\Pi}$ can be computed numerically following the procedure developed by Aravas and Ponte Castañeda (2004). Similar to the strain-rate concentration tensor, the spin concentration tensor $\mathbb{C}_{[1]}^{(2)}$ corresponding to the first-level LCC, depends on the shape and orientation of both the pores and distribution; the other spin concentration tensors $\mathbb{C}_{[i]}^{(2)}(i=2, \ldots, N)$ corresponding to higher-level LCCs, depend only on the shape and orientation of the pore distribution.

Given the above expressions (33), (34), (35), along with relation (13), the plastic spin 
$\overline{\mathbf{W}}^{p}=\overline{\mathbf{W}}-\overline{\boldsymbol{\omega}}$ can be given by

$$
\begin{aligned}
\overline{\mathbf{W}}^{p}= & -\frac{1}{2} \sum_{\substack{\alpha, \beta=1 \\
\alpha \neq \beta \\
w_{\alpha} \neq w_{\beta}}}^{3} \frac{w_{\alpha}^{2}+w_{\beta}^{2}}{w_{\alpha}^{2}-w_{\beta}^{2}}\left[\left(\mathbf{n}_{\alpha} \otimes \mathbf{n}_{\beta}+\mathbf{n}_{\beta} \otimes \mathbf{n}_{\alpha}\right) \cdot\left(\prod_{i=1}^{N} \mathbb{A}_{[i]}^{(2)}\right) \overline{\mathbf{D}}\right] \mathbf{n}_{\alpha} \otimes \mathbf{n}_{\beta} \\
& +\sum_{i=1}^{N}\left[\mathbb{C}_{[i]}^{(2)}\left(\prod_{j=i+1}^{N} \mathbb{A}_{[j]}^{(2)}\right)\right] \overline{\mathbf{D}} .
\end{aligned}
$$

Consistent with the work of Aravas and Ponte Castañeda (2004), the plastic spin may then be rewritten as

$$
\overline{\mathbf{W}}^{p}=\dot{\lambda} \bar{\Omega}^{p}
$$

where $\bar{\Omega}^{p}$ is given by

$$
\begin{aligned}
\overline{\mathbf{\Omega}}^{p}= & -\frac{1}{2} \sum_{\substack{\alpha, \beta=1 \\
\alpha \neq \beta \\
w_{\alpha} \neq w_{\beta}}}^{3} \frac{w_{\alpha}^{2}+w_{\beta}^{2}}{w_{\alpha}^{2}-w_{\beta}^{2}}\left[\left(\mathbf{n}_{\alpha} \otimes \mathbf{n}_{\beta}+\mathbf{n}_{\beta} \otimes \mathbf{n}_{\alpha}\right) \cdot\left(\prod_{i=1}^{N} \mathbb{A}_{[i]}^{(2)}\right) \mathbf{N}\right] \mathbf{n}_{\alpha} \otimes \mathbf{n}_{\beta} \\
& +\sum_{i=1}^{N}\left[\mathbb{C}_{[i]}^{(2)}\left(\prod_{j=i+1}^{N} \mathbb{A}_{[j]}^{(2)}\right)\right] \mathbf{N}
\end{aligned}
$$

\section{References}

[1] Agoras, M., Ponte Castañeda, P., 2011. Homogenization estimates for multi-scale nonlinear composites. Eur. J. Mech. A/Solids. 30, 828-843.

[2] Agoras, M., Ponte Castañeda, P., 2013. Iterated linear comparison bounds for viscoplastic porous materials with "ellipsoidal" microstructures. J. Mech. Phys. Solids. 61, 701-725.

[3] Agoras, M., Ponte Castañeda, P., 2014. Anisotropic finite-strain models for porous viscoplastic materials with microstructure evolution. Int. J. Solids Struct. 51, 981-1002.

[4] Aravas, N., Ponte Castañeda, P., 2004. Numerical methods for porous metals with deformation-induced anisotropy. Comput. Methods Appl. Mech. Engrg. 193, 3767-3805.

[5] Bao, Y., Wierzbicki, T., 2004. On fracture locus in the equivalent strain and stress triaxiality space. Int. J. Mech. Sciences 46, 81-98. 
[6] Barsoum, I., Faleskog, J., 2007. Rupture mechanisms in combined tension and shearExperiments. Int. J. Solids Struct. 44, 1768-1786.

[7] Benzerga, A.A., Leblond, J-B., 2010. Ductile fracture by void growth to coalescence. Adv. Appl. Mech. 44, 169-305.

[8] Dafalias, Y.F.., 1985. The plastic spin. J.. Appl. Mech. 52, 865871.

[9] Danas, K., Idiart, M.I., Ponte Castañeda, P., 2008. A homogenization-based constitutive model for isotropic viscoplastic porous media. Int. J. Solids Struct. 45, 3392-3409.

[10] Danas, K., Ponte Castañeda, P., 2009a. A finite-strain model for anisotropic viscoplastic porous media: I-Theory. Eur. J. Mech. A/Solids 28, 387-401.

[11] Danas, K., Ponte Castañeda, P., 2009b. A finite-strain model for anisotropic viscoplastic porous media: II-Applications. Eur. J. Mech. A/Solids 28, 402-416.

[12] Danas, K., Ponte Castañeda, P., 2012. Influence of the Lode parameter and the stress triaxiality on the failure of elasto-plastic porous materials. Int. J. Solids Struct. 49, $1325-1342$.

[13] Gologanu, M., Leblond, J.-B., Devaux, J., 1993. Approximate models for ductile metals containing non-spherical voids - case of axisymmetric prolate ellipsoidal cavities. J. Mech. Phys. Solids 41, 1723-1754.

[14] Gurson, A. L., 1977. Continuum theory of ductile rupture by void nucleation and growth. J. Engng. Mater. Technol. 99, 2-15.

[15] Haltom, S.S., Kyriakides, S., Ravi-Chandar, K., 2013. Ductile failure under combined shear and tension. Int. J. Solids Struct. 50, 1507-1522.

[16] Hutchinson, J. W., Tvergaard, V., 2012. Comment on "Influence of the Lode parameter and the stress triaxiality on the failure of elasto-plastic porous materials" by K. Danas and P. Ponte Castañeda. Int. J. Solids Struct. 49, 3484-3485.

[17] Idiart, M.I., 2008. Modeling the macroscopic behavior of two-phase nonlinear composites by infinite-rank laminates. J. Mech. Phys. Solids 56, 2599-2617. 
[18] Idiart, M. I., Ponte Castañeda, P, 2007. Field statistics in nonlinear composites. I. Theory. Proc. R. Soc. Lond. A 463, 183-202.

[19] Kailasam, M., 1998. A general constitutive theory for particulate composites and porous materials with evolving microstructures, Ph.D. thesis, University of Pennsylvania, 1998.

[20] Kailasam, M., Aravas, N., Ponte Castañeda, P., 2000. Porous metals with developing anisotropy: Constitutive models, computational issues and applications to deformation processing. Computer Modeling in Engineering and Sciences 1, 105-118.

[21] Kailasam, M., Ponte Castañeda, P., 1997. The evolution of anisotropy in porous materials and its implications for shear localization. IUTAM Symposium on Mechanics of Granular and Porous Materials, N.A. Fleck and A.C.F. Cocks, Eds., Kluwer Academic Publishers, 365-376.

[22] Kailasam, M., Ponte Castañeda, P., 1998. A general constitutive theory for linear and nonlinear particulate media with microstructure evolution. J. Mech. Phys. Solids 46, $427-465$.

[23] Kailasam, M., Ponte Castañeda, P., Willis, J. R., 1997. The effect of particle size, shape, distribution and their evolution on the constitutive response of nonlinearly viscous composites. I. Theory. Phil. Trans. R. Soc. Lond. A 355, 1835-1852.

[24] Lebensohn, R.A., Idiart, M.I., Ponte Castañeda, P. Vincent, P.-G., 2011. Dilatational viscoplasticity of polycrystalline solids with intergranular cavities. Phil. Mag. 91, 30383067.

[25] Leblond, J-B., Perrin, G., Suquet, P., 1994. Exact results and approximate models for porous viscoplastic solids. Int. J. Plasticity 10, 213-235.

[26] Madou, K., Leblond, J-B., 2012a. A Gurson-type criterion for porous ductile solids containing arbitrary ellipsoidal voids-I: Limit-analysis of some representative cell. J. Mech. Phys. Solids 60, 1020-1036. 
[27] Madou, K., Leblond, J-B., 2013a. Numerical studies of porous ductile materials containing arbitrary ellipsoidal voids - I: Yield surfaces of representative cells. Eur. J. Mech. A/Solids 42, 480-489.

[28] Madou, K., Leblond, J-B., Morin, L., 2013b. Numerical studies of porous ductile materials containing arbitrary ellipsoidal voids - II: Evolution of the length and orientation of the void axes. Eur. J. Mech. A/Solids 42, 490-507.

[29] Michel, J.C., Suquet, P., 1992. The constitutive law of nonlinear viscous and porous materials. J. Mech. Phys. Solids 40, $783-812$.

[30] Nahshon, K., Hutchinson, J. W., 2008. Modification of the Gurson model for shear failure. Eur. J. Mechanics A/Solids 27, 1-17.

[31] Needleman, A., 1972. Void growth in an elastic-plastic medium. Journal of Applied Mechanics 39, 964-970.

[32] Ogden, 1984. Non-linear elastic deformations. Halsted Press, New York 128-130

[33] Ponte Castañeda, P., 1991. The effective mechanical properties of nonlinear isotropic composites. J. Mech. Phys. Solids 39, 45-71.

[34] Ponte Castañeda, P., 2002. Second-order homogenization estimates for nonlinear composites incorporating field fluctuations. I - Theory. J. Mech. Phys. Solids 50, 737-757.

[35] Ponte Castañeda, P., 2012. Bounds for nonlinear composites via iterated homogenization. J. Mech. Phys. Solids 60, 1583-1604.

[36] Ponte Castañeda, P., Suquet, P., 1998. Nonlinear composites. Adv. Appl. Mech. 34, $171-302$.

[37] Ponte Castañeda, P., Willis, J. R., 1988. On the overall properties of nonlinearly viscous composites. Proc. R. Soc. Lond. A 416, 217-244.

[38] Ponte Castañeda, P., Willis, J. R., 1995. The effect of spatial distribution on the effective behavior of composite materials and cracked media. J. Mech. Phys. Solids 43, $1919-1951$. 
[39] Ponte Castañeda, P., Zaidman, M., 1994. Constitutive models for porous materials with evolving microstructures. J. Mech. Phys. Solids 42, 1459-1497.

[40] Rice, J.R., 1977. The localization of plastic deformation. Proceedings of the 14th International Congress of Theoretical and Applied Mechanics, North-Holland Publishing Company, pp. 207-220.

[41] Srivastava, A., Needleman, A., 1977. Void growth versus void collapse in a creeping single crystal. Journal of the Mechanics and Physics of Solids 61, 1169-1184.

[42] Talbot, D.R.S.., Willis, J. R., 1985. Variational principles for inhomogeneous non-linear media. I.M.A. J. appl. Math. 35, 39-54.

[43] Tvergaard, V., 1981. Influence of voids on shear band instabilities under plane strain conditions. Int. J. Fracture 17, 389-407.

[44] Tvergaard, V., 1990. Material failure by void growth. Adv. Appl. Mech. 27, 83-151.

[45] Tvergaard, V., 2012. Effect of stress-state and spacing on voids in a shear-field. International Journal of Solids and Structures 49, 3047-3054..

[46] Tvergaard, V., 2014. Bifurcation into a localized mode from non-uniform periodic deformations around a periodic pattern of voids. J. Mech. Phys. Solids 69, 112-122.

[47] Yamamoto, H., 1978. Conditions for shear localization in the ductile fracture of void containing materials. Int. J. Fract. 14, 347-365.

[48] Willis, J. R., 1977. Bounds and self-consistent estimates for the overall moduli of anisotropic composites. J. Mech. Phys. Solids 25, 185-202.

[49] Willis, J.R., 1991. On methods for bounding the overall properties of nonlinear composites. J. Mech. Phys. Solids 39, 73-86.

[50] Xue, Z,. Faleskog, J., Hutchinson, J. W., 2013. Tension-torsion fracture experiments Part II: Simulations with the extended Gurson model and a ductile fracture criterion based on plastic strain. Int. J. Solids Struct. 50, 4258-4269. 